\title{
Delayed-feedback control: arbitrary and distributed delay-time and noninvasive control of synchrony in networks with heterogeneous delays
}

\author{
Chol-Ung Choe • Ryong-Son Kim • Hyok Jang • \\ Philipp Hövel · Eckehard Schöll
}

Received: 6 November 2013 / Revised: 2 December 2013 / Accepted: 2 December 2013 / Published online: 4 January 2014

(C) Springer-Verlag Berlin Heidelberg 2014

\begin{abstract}
We suggest a delayed feedback control scheme with arbitrary delay for stabilizing a periodic orbit, while maintaining the noninvasiveness of the controller. Since the constraint on the delay to be adjusted to the period of the unstable periodic orbit is not imposed, a richer structure of the dynamics can be observed: Not only weakly unstable, but also strongly unstable periodic orbits are stabilized and even stabilization of orbits with infinite period is achieved. The control mechanism is elucidated for the generic model of a subcritical Hopf bifurcation. A complete bifurcation analysis for the fixed point as well as the periodic orbit is presented and the stability domains are identified. Furthermore, we study the effects of distributed delayed feedback on the stabilization of periodic orbits, and show that larger variance of the delay distribution considerably enlarges the stabilization region in the parameter space. We extend the control scheme to a network of Hopf normal forms coupled with heterogeneous delays. By tuning the coupling parameters, different synchronization patterns, i.e., in-phase, splay, and clustering, can be selected. The characteristic equation for Floquet exponents of the heterogeneous delay network is derived in an analytical form, which reveals the coupling parameters for successful stabilization. The equation takes a
\end{abstract}

C.-U. Choe $\cdot$ R.-S. Kim $\cdot$ H. Jang

Center for Nonlinear Science, University of Science,

Unjong-District, Pyongyang, DPR Korea

P. Hövel · E. Schöll $(\bowtie)$

Institut für Theoretische Physik, Technische Universität Berlin,

Hardenbergstraße 36, 10623 Berlin, Germany

e-mail: schoell@physik.tu-berlin.de

P. Hövel

Bernstein Center for Computational Neuroscience,

Humboldt-Universität zu Berlin, Philippstraße 13,

10115 Berlin, Germany unified form for both subcritical and supercritical Hopf bifurcations regardless of the synchronization patterns. Analysis of Floquet exponents and direct numerical simulations show that the heterogeneity in the delays drastically facilitates stabilization and provides an enlarged parameter region for successful control. Finally, we consider the thermodynamic limit in the framework of a mean field approximation and show that heterogeneous delays offer an enhanced performance of control.

Keywords Time-delayed feedback control . Synchronization · Networks · Heterogeneous delay · Hopf normal form

\section{Introduction}

Time delayed feedback control (DFC), proposed by Pyragas [1], is a simple and convenient method to stabilize unstable periodic orbits (UPOs) occurring in a single dynamical system. Since DFC uses only the difference of the current and the delayed state, where the time delay is given by the period of the UPO, the control is noninvasive. Furthermore, the control is applicable to systems whose equations of motion are unknown. Due to this convenience, the algorithm of DFC has been applied to quite diverse experimental systems, and many theoretical advances have also been made [2-5]. However, it was commonly believed that torsion-free UPOs cannot be stabilized by DFC [6]. To overcome this limitation, modified control schemes, like an oscillating feedback [7], a half-period delay [8] and the introduction of an unstable controller [9-11] were proposed.

This alleged odd-number theorem has been refuted [12] by a counterexample, using the normal form of a subcritical Hopf bifurcation that is a representative system for the odd- 
number limitation. The normal form of a sub- or supercritical Hopf bifurcation is also called Stuart-Landau oscillator, and is often used as a generic model for oscillators. Experimental evidence for the predicted DFC of odd-number orbits generated by a subcritical Hopf bifurcation has recently been presented $[13,14]$. For a state-of-the-art general discussion of the odd-number limitation see Ref. $[15,16]$. The normal form of a system near a subcritical Hopf bifurcation also allows for an analytical treatment of the stability of the UPO, including the calculation of the Floquet exponents [17-20]. However, since the constraint is imposed that the delay time should be adjusted to the period of the UPO, strongly unstable periodic orbits are difficult to stabilize.

Meanwhile, coupled systems ranging from a few elements to large networks have become a central issue in nonlinear science [21-24]. In particular, the study of the synchronization of networks has evolved into a rapidly expanding field $[25,26]$. Time delays are always present in coupled systems due to the finite signal propagation time. These time lags give rise to complex dynamics and have been shown to play a key role in the synchronization behavior of systems [27-42], see also the review [43]. In Ref. [20,44,45], we have shown that UPOs in networks of Hopf normal forms can be stabilized to exhibit in-phase synchronization in the sub- and supercritical case, and that additionnally more general splay or cluster synchronization patterns can be stabilized in the supercritical case. Here we extend these results in various ways; among other issues, we show that cluster synchronization can also be stabilized in networks of subcritical Hopf normal forms, and that one can deliberately switch in a unique and noninvasive form, including the case of heterogeneous delays, between different synchronous states in a network of supercritical Hopf normal forms by adjusting the coupling phase parameter.

In practice, the exact values of the system parameters might be unknown, and they might be heterogeneous, and moreover, one cannot always adjust the delays to a specified value, e.g., to the period of the local node dynamics. In particular, it has been pointed out in the biological context that heterogeneous delays lead to more realistic models [46]. Nevertheless, most studies have assumed that all the interactions occur with the same time delay and, up to now, little is known about stabilizing UPOs and controlling the synchrony patterns in networks coupled with heterogeneous delays. For instance, the dynamics of an array of chaotic logistic maps coupled with random delay times [47], the effects of heterogeneous delays in the coupling of two excitable neural systems $[48,49]$ or a neural network [50], and amplitude death in the Stuart-Landau system coupled with distributed delays [51-53] or periodically modulated delay [54] were investigated.

In this paper, we suggest a DFC scheme with arbitrary delay for stabilizing a UPO, but maintain the noninvasiveness property, for the normal form of a subcritical Hopf bifurcation. Unlike the conventional DFC, where the delay-time is adjusted to match the period of the target orbit, a rotation of the feedback term is used to obtain noninvasive control. Since the constraint on the delay to be adjusted to the period is not imposed in the present study, rich dynamics can be found for different delays. A complete bifurcation analysis for the fixed point as well as for the periodic orbit is presented, and the stability domains are identified. We will show that both weakly and strongly unstable periodic orbits can be stabilized. The former have a global basin of attraction and the latter have only a local basin. Furthermore, stabilization of orbits with infinite period is possible. We also introduce a nonlinear feedback control scheme, which is more efficient than the linear one, with respect to both the control aim and the theoretical analysis, and discuss effects of distributed delays in the feedback.

We extend the feedback control scheme to a network of Hopf normal forms coupled with heterogeneous delays. By tuning the coupling rotations, only one state among different synchronization patterns, i.e., in-phase, splay, and clustering, can be chosen in noninvasive form and their stabilizations can be efficiently achieved. Adopting the rotation of the delay terms as a control parameter, rather than the coupling phase, makes it possible to remove multistabilities of the collective amplitude and frequency that were undesirable effects in the previous studies $[44,45]$. Our control scheme might be viewed as a natural way to model the network coupled with delay proportional to the distance and with rotation of the input signal proportional to the distance. They could thus be particularly suitable for networks of semiconductor lasers and in neuroscience. Similar extensions of the Pyragas control scheme based on the symmetry-breaking of equivariant Hopf bifurcation $[55,56]$ have recently been suggested and applied to noninvasive and pattern-selective stabilization of three coupled Stuart-Landau oscillators (ponies on a merrygo-round) [55].

The characteristic equation for Floquet exponents of the heterogeneous delay network is derived in an analytical form, which reveals the coupling parameters for successful stabilization. The equation takes a unified form regardless of the Hopf bifurcation forms including the subcritical and supercritical case and regardless of the synchronization patterns. As a result we can show that UPOs (limit cycles) in many types of networks can be stabilized in cluster form by heterogeneous delayed coupling. Our results of controlling the periodic orbits are demonstrated also by direct numerical simulations. The effects of heterogeneous delays on the cluster stabilization of periodic orbits in noninvasive form are discussed, and it is shown that the heterogeneity in the delays can greatly enlarge the set of parameters for successful control. We consider the thermodynamic limit of the network system in the frame of mean-field approximation, and derive 
the characteristic equations for the stability of UPOs, relating them to the distribution of the heterogeneous delays. Our results show that heterogeneous delays can greatly enhance the control of UPOs.

The paper is organized as follows. In Sect. 2 we consider delayed-feedback control with arbitrary delay for a single Hopf normal form. Two coupling schemes, namely linear and nonlinear, for DFC of the normal form of a subcritical Hopf bifurcation are proposed in Sect. 2.1. Sections 2.2, 2.3 and 2.4 are devoted to a complete bifurcation analysis for the fixed point as well as the periodic orbit. Section 3 describes the effect of distributed delays. In Sect. 4, we consider networks of Hopf normal forms coupled with heterogeneous delays and provide the example of a bidirectional ring in Sect. 4.1. The characteristic equations for Floquet exponents of networks coupled with heterogeneous delays are derived and the effects of heterogeneous delays on the clustering are presented in Sect. 4.2. Section 4.3 describes the special cases and the numerical results. In Sect. 4.4, the thermodynamic limit in the framework of a mean-field approximation is considered. Finally, we conclude with Sect. 5. In the Appendix, the normal form of a Hopf bifurcation of the fixed point is constructed using the method of multiple scales.

\section{Self-feedback control with arbitrary delay in a single system}

Consider the stabilization of a UPO with period $T, \mathbf{x}_{0}(t-$ $T)=\mathbf{x}_{0}(t)$, contained in a dynamical system

$\dot{\mathbf{x}}=\mathbf{F}(\mathbf{x})$,

where $\mathbf{x} \in \mathbb{R}^{n}$ and $\mathbf{F}$ is a nonlinear function. If there exists an operator $S(t ; \tau)$, represented by a matrix, that satisfies $[55,57,58]$

$S(t ; \tau) \mathbf{x}_{0}(t-\tau)=\mathbf{x}_{0}(t)$,

one can consider, instead of the original Pyragas method [1], a control scheme with arbitrary delay time $\tau$ as follows:

$\dot{\mathbf{x}}=\mathbf{F}(\mathbf{x})+\mathbf{K}[S(t ; \tau) \mathbf{x}(t-\tau)-\mathbf{x}(t)]$,

where $\mathbf{K}$ is a feedback gain matrix. Equation (2) is reduced to the Pyragas control scheme for $\tau=T$ since $S(t ; T)$ becomes the identity matrix.

By construction, the control scheme given by Eq. (2) is noninvasive: the control force vanishes at successful stabilization, and the periodic orbit itself remains untouched by the control terms. In general, however, it is difficult to find a transformation matrix $S(t, \tau)$ satisfying Eq. (1) unless full information of the UPO is given. If we consider, however, the normal form for Hopf bifurcation, then we can overcome this limitation by taking the $S^{1}$ equivariance of the system into account.

\subsection{Time-delayed feedback scheme}

As a generic model system for time-delayed feedback control of periodic orbits, we consider the normal form of the subcritical Hopf bifurcation. The equation of motion subjected to a delayed feedback control with an unspecified (arbitrary) delay time $\tau$ reads

$$
\begin{aligned}
\dot{z}(t)= & {\left[\lambda+i \omega_{0}+(1+i \gamma)|z(t)|^{2}\right] z(t) } \\
& +K e^{i \beta}[w(t ; \tau) z(t-\tau)-z(t)],
\end{aligned}
$$

with the complex variable $z=r e^{i \theta} \in \mathbb{C}, \lambda, \omega_{0}, \gamma \in \mathbb{R}$. For simplicity, the feedback gain matrix $\mathbf{K}$ of Eq. (2) is modeled as complex number $K e^{i \beta}$ with feedback strength $K$ and phase $\beta$. In the absence of feedback $(K=0)$, a UPO with the radius $r=r_{0} \equiv \sqrt{-\lambda}$ and period $T=2 \pi /\left(\omega_{0}-\gamma \lambda\right)$, i.e., angular frequency $\Omega_{0}=\omega_{0}-\gamma \lambda$, exists for $\lambda<0$. The control operator $S(t ; \tau)$ of Eq. (1) is given by the complex function $w(t ; \tau)=|w| e^{i \psi(\tau)}$, and we suggest two types of of feedback: (i) a linear control force using

$w=w_{L} \equiv e^{i \psi(\tau)}$

and (ii) a nonlinear one using

$w=w_{N L} \equiv \frac{|z(t)|}{|z(t-\tau)|} e^{i \psi(\tau)}$,

where the rotational part is given by $\psi(\tau)=\Omega_{0} \tau$ in order to compensate the time delay $\tau$.

We stress that both choices Eqs. (4) and (5) guarantee that the target UPO is reproduced automatically and noninvasively for any delay time $\tau$ by construction of the control system (3). This is in contrast to the conventional DFC, in which the delay time should be adjusted to the period of the UPO, $\tau=T$, defining the Pyragas curve

$\tau=\frac{2 \pi}{\omega_{0}-\gamma \lambda}$

In our study, we will no longer impose the constraint (6). Furthermore, the inequality $\gamma \lambda<\omega_{0}$ can be relaxed. This implies that the rotations around the fixed point and UPO could be in opposite direction and that strongly unstable orbits are also feasible as target UPO involved. Moreover, the extreme case, $\gamma \lambda=\omega_{0}$, at which the period of the UPO becomes infinite, is not excluded from our considerations.

Throughout our analytical study the coupling phase $\beta$ is confined to an interval of $[0, \pi / 2]$ and the control amplitude $K$ takes positive values or, equivalently, the $K$ values satisfy $K \cos \beta>0$ with $\beta \in[0, \pi]$. In our numerical diagrams 
we will usually fix the three parameters, $\omega_{0}, \gamma$, and $\beta$, to $\omega_{0}=1, \gamma=-10$, and $\beta=\pi / 4$.

Obviously, the linear feedback system, Eqs. (3) and (4), is reduced to Pyragas control when the delay time $\tau$ coincides with the period $T$ since $e^{i \Omega_{0} T}=1$. On the other hand, the choice in Eq. (5) means that the delayed-feedback in Eq. (3) might still be influenced by the phase of the variable, but not by the amplitude. Nevertheless, it is worth to mention that with such a choice the parameter region for successful control becomes remarkably larger than with the linear technique and, moreover, a thorough analytical study for the stabilization of the UPO becomes possible, as will be shown in Sect. 2.3.

\subsection{Stability analysis of the fixed point}

Stabilization of the UPO does not necessarily require destabilization of the fixed point. There could, for instance, be a parameter range with multistability in the presence of an additional delay-induced orbit. However, the stabilization could be accelerated and achieved with a larger basin of attraction, i.e., the whole region inside the UPO. Moreover, the properties of the two schemes are closely related since the feedback term includes the frequency of the UPO.

\subsubsection{Hopf curves of the fixed point}

The exponential ansatz $z=\exp (\eta t)$ yields a transcendental characteristic equation for evaluating the stability of the trivial fixed point $z_{*}=0: \eta=\lambda+i \omega_{0}+$ $K e^{i \beta}\left(e^{-\eta \tau+i \Omega_{0} \tau}-1\right)$, or, equivalently, using $\eta-i \Omega_{0}=\bar{\eta}$,

$\bar{\eta}=\lambda(1+i \gamma)+K e^{i \beta}\left(e^{-\bar{\eta} \tau}-1\right)$.

Note that the coefficient of the cubic term of the Hopf normal system, $(1+i \gamma)$, appears in Eq. (7) for the linear stability analysis of the fixed point. Thus, it is not surprising that the characteristic equations for the fixed point and the UPO are closely related to each other.

A Hopf bifurcation of the fixed point that occurs for $\bar{\eta}=$ $i \omega_{H}$ gives

$i \omega_{H} \tau=\lambda(1+i \gamma)+K e^{i \beta}\left(e^{-i \omega_{H} \tau}-1\right)$,

which yields the Hopf curves, or more precisely, the Hopf surface in the $(\lambda, \tau, K)$-parameter space with parametric representation

$\lambda=K[\cos \beta-\cos (\beta-\phi)]$,

$\tau=\frac{\phi}{\gamma \lambda+K[\sin (\beta-\phi)-\sin \beta]}$, where $\phi=\omega_{H} \tau$. In other words, separating Eq. (8) into real and imaginary parts

$-\lambda=K\left[\cos \left(\beta-\omega_{H} \tau\right)-\cos \beta\right]$,

$\omega_{H}-\gamma \lambda=K\left[\sin \left(\beta-\omega_{H} \tau\right)-\sin \beta\right]$,

and using trigonometric identity $\left[\cos \left(\beta-\omega_{H} \tau\right)\right]^{2}+[\sin$ $\left.\left(\beta-\omega_{H} \tau\right)\right]^{2}=1$, the Hopf frequency is determined by

$\omega_{H}=\omega_{ \pm} \equiv \gamma \lambda-K \sin \beta \pm \sqrt{\Delta}$,

where $\Delta=K^{2}-(K \cos \beta-\lambda)^{2}$. Note that the Hopf frequency is defined under the condition $\Delta \geq 0$, i.e.,

$K(\cos \beta-1) \leq \lambda<0$.

Equations $(10 a, b)$ can be rewritten as

$K \cos \left(\omega_{ \pm} \tau-\beta\right)=K \cos \beta-\lambda$,

$K \sin \left(\omega_{ \pm} \tau-\beta\right)=\mp \sqrt{\Delta}$.

Since the right-hand side of Eq. (12a) is always positive for $\lambda<0$, the principal values of $\omega_{+} \tau-\beta$ and $\omega_{-} \tau-\beta$ lie in the intervals $(-\pi / 2,0)$ and $(0, \pi / 2)$, respectively, i.e., $0<\arccos (\cos \beta-\lambda / K)<\pi / 2$ and

$\omega_{+} \tau-\beta=-\theta+2 n \pi$,

$\omega_{-} \tau-\beta=\theta+2 n \pi$,

where $n=0, \pm 1, \pm 2, \ldots$ and $\theta=\arccos (\cos \beta-\lambda / K)$. The inequality $\theta<\beta$ holds for $\lambda<0$, since $\sin \theta=$ $\sqrt{\sin ^{2} \beta+2(\lambda / K) \cos \beta-\lambda^{2} / K^{2}}<\sin \beta$.

Summarizing these results, we obtain an explicit expression for the multi-valued Hopf curve for a given control amplitude $K$ and $\lambda$ :

$$
\begin{aligned}
\tau_{n}^{+} & =\frac{-\theta+\beta+2 n \pi}{\omega_{+}} \\
& =\frac{-\arccos (\cos \beta-\lambda / K)+\beta+2 n \pi}{\gamma \lambda-K \sin \beta+\sqrt{K^{2}-(K \cos \beta-\lambda)^{2}}}, \\
\tau_{n}^{-} & =\frac{\theta+\beta+2 n \pi}{\omega_{-}} \\
& =\frac{\arccos (\cos \beta-\lambda / K)+\beta+2 n \pi}{\gamma \lambda-K \sin \beta-\sqrt{K^{2}-(K \cos \beta-\lambda)^{2}}} .
\end{aligned}
$$

It is clear that two Hopf curves, $\tau_{n}^{+}$and $\tau_{n}^{-}$, meet at points $A_{n}$ for $n=0,1,2, \ldots$, satisfying the relation $(\lambda, \tau)=\left(K(\cos \beta-1), \frac{\beta+2 n \pi}{K[\gamma(\cos \beta-1)-\sin \beta]}\right)$, or equivalently, $(K, \tau)=\left(\frac{\lambda}{\cos \beta-1}, \frac{(\cos \beta-1)(\beta+2 n \pi)}{\lambda[\gamma(\cos \beta-1)-\sin \beta]}\right)$.

Note that we assumed $\omega_{ \pm} \neq 0$ in the derivation of the Hopf curves (14a). However, one can see in Eq. (13a) the following relations

$$
\begin{aligned}
& \left.\omega_{+}\right|_{\lambda=0}=0, \\
& \left.\omega_{-}\right|_{\lambda=\lambda_{\perp}}=0,
\end{aligned}
$$


where $\lambda_{\perp}=2 K(\gamma \sin \beta-\cos \beta) /\left(1+\gamma^{2}\right)<0$. For the case of Eq. (15a), in particular, Eq. (10a) holds for any $\tau$. Thus, $\lambda=0$, i.e., the $\tau$ axis in the $(\lambda, \tau)$-plane also forms another part of Hopf curves besides the curves given by Eq. (14a) (see the lines denoted by $\mathrm{H}^{+}$and $\mathrm{H}^{-}$in Fig. 1b).

None of the Hopf branches determined by Eq. (14a) emanates from the original point $(\lambda, \tau)=(0,0)$, and the crossings of the branches along the $\tau$ axis are given by

$\omega_{+} \tau_{n}^{+}=2 n \pi$,

$\omega_{-} \tau_{n}^{-}=2 \beta+2 n \pi$

with $\omega_{+}=0$ and $\omega_{-}=-2 K \sin \beta$ for $\lambda=0$. In Eq. (16a), the case $n=0$ makes sense only for a definite value of $\tau_{0}^{+}$ since $\omega_{+}=0$, in which $\tau_{0}^{+}$takes the value $0 / 0$. As $\lambda \rightarrow 0$, the intersection is determined by.

$\tau_{0}^{+}=\lim _{\lambda \rightarrow 0} \frac{-\theta+\beta}{\omega_{+}}=\frac{-1}{K(\cos \beta+\gamma \sin \beta)}$.

As a result, we obtain the crossing points $B_{-n}$ given by

$\tau_{0}^{B}=\tau^{*}$,

$\tau_{n}^{B}=-\frac{\beta+n \pi}{K \sin \beta}$,

where $\tau^{*}=-1 / K(\cos \beta+\gamma \sin \beta)$ and $n=-1,-2, \ldots$. The integers $n$ in Eq. (18a) have to take negative values in order to satisfy the inequality $\tau_{n}^{B}>0$ for $\beta \in(0, \pi)$ and negative values of $\gamma$. Thus, for the positive branches $\tau_{n}^{+}$, the crossing of Hopf curves with $\tau$ axis occurs only at the point $\tau^{*}$, and other crossing points are generated by negative branches $\tau_{n}^{-}$with negative $n$ (see Fig. 1a).

\subsubsection{Codimension-2 bifurcations on Hopf curves}

Next, we evaluate the crossing directions of the critical Hopf eigenvalues on the Hopf curves. Abbreviating $\frac{\partial}{\partial \tau} \bar{\eta}$ by $\bar{\eta}_{\tau}$ the crossing direction for the $\tau$ axis direction is given by $\operatorname{sign}\left(\operatorname{Re} \bar{\eta}_{\tau}\right)$. Implicit differentiation of Eq. (7) with respect to $\tau$ gives

$\bar{\eta}_{\tau}=\frac{-K \bar{\eta} e^{i \beta-\bar{\eta} \tau}}{1+K \tau e^{i \beta-\bar{\eta} \tau}}$,

and

$\left.\operatorname{Re} \bar{\eta}_{\tau}\right|_{\bar{\eta}=i \omega_{ \pm}}= \pm \frac{\omega_{ \pm} \sqrt{\Delta}}{|D|^{2}}$

where $\Delta=K^{2}-(K \cos \beta-\lambda)^{2}>0$ and $D=1+K \tau e^{i \beta-\bar{\eta} \tau}$. Taking into account that $\left.\omega_{+}\right|_{\lambda=K(\cos \beta-1)}=-2 K \sin \frac{\dot{\beta}}{2}$ $\left(\gamma \sin \frac{\beta}{2}+\cos \frac{\beta}{2}\right)>0$ for $\gamma<-\cot \frac{\beta}{2}$, and $\omega_{+}=0$ if and only if $\lambda=0$, then it turns out that $\omega_{+}$takes always the positive values for $\lambda<0$ and $\gamma<-\cot \frac{\beta}{2}$. Thus, Eq. (19) yields

$\operatorname{Re}\left(\bar{\eta}_{\tau} \bar{\eta}_{\bar{\eta}=i \omega_{+}}\right) \begin{cases}>0, & \text { for } \lambda<0 \\ <0, & \text { for } \lambda>0 .\end{cases}$

On the other hand, $\omega_{-}$is decreasing with the increasing $\lambda$ due to

$\frac{d \omega_{-}}{d \lambda}=\gamma-\frac{K \cos \beta-\lambda}{\sqrt{\Delta}}<0$,

which results in the fact that $\omega_{-}>0$ for $\lambda<\lambda_{\perp}$ and $\omega_{-}<0$ for $\lambda>\lambda_{\perp}$ since $\omega_{-}=0$ at $\lambda=\lambda_{\perp}$. Therefore,

$\operatorname{Re}\left(\left.\bar{\eta}_{\tau}\right|_{\bar{\eta}=i \omega_{-}}\right) \begin{cases}<0, & \text { for } \lambda<\lambda_{\perp} \\ >0, & \text { for } \lambda>\lambda_{\perp} .\end{cases}$

As a result, we see that the Hopf curves $\tau_{n}^{+}$correspond to destabilization of the fixed point for the $\tau$ axis direction when $\lambda<0$, while $\tau_{n}^{-}$correspond to stabilization for the same direction when $\lambda<\lambda_{\perp}$. The primary branch $\tau_{0}^{+}$for $\lambda>0$ and the curves $\tau_{n}^{-}$with the negative $n$ for $\lambda>\lambda_{\perp}$ represent the boundaries for the stabilization and destabilization for the $\tau$ axis direction, respectively.

The region for the destabilization of the fixed point can be described as follow as:

$\tau_{n}^{+}<\tau<\tau_{n}^{-}$for $\lambda<0$ and $n \geq 0$,

$\tau>\tau_{n}^{-}$for $\lambda>\lambda_{\perp}$ and $n<0$

$\tau<\tau_{0}^{+}$for $\lambda>0$.

The crossing points $A_{n}$ of Hopf curves, $\tau_{n}^{+}$and $\tau_{n}^{-}$, are codimension-2 bifurcation points, but do not coincide with the transition point from sub- to supercritical Hopf bifurcation. The criterion for the super- and subcritical behavior can be derived by using the multiple scales method as follows (see Appendix):

$$
\begin{aligned}
1 & +K \tau\left[\cos \left(\beta+\Omega_{0} \tau-\phi\right)\right. \\
& \left.+\gamma \sin \left(\beta+\Omega_{0} \tau-\phi\right)\right] \begin{cases}<0, & \text { supercritical, } \\
>0, & \text { subcritical. }\end{cases}
\end{aligned}
$$

Here, the control amplitude $K$, the delay time $\tau$ and $\lambda$ are to be determined by Eq. (9a). Equation (24) gives the value of $\phi$ for the transition point from sub- to supercritical Hopf bifurcation. Since $\Omega_{0} T=2 \pi$ for the choice $\tau=T$, Eq. (24) coincides with that obtained in [17], in which approximation of the center manifold was used for determining the criterion for the super- and subcritical behavior.

In a similar way the crossing directions of the critical Hopf eigenvalues on the other Hopf curve, i.e., $\tau$ axis, can be determined. Abbreviating $\frac{\partial}{\partial \lambda} \bar{\eta}$ by $\bar{\eta}_{\lambda}$, the crossing direction for the $\lambda$-axis direction is given by $\operatorname{sign}\left(\operatorname{Re} \bar{\eta}_{\lambda}\right)$. Implicit differentiation of Eq. (7) with respect to $\lambda$ at $\lambda=0$ and $\omega_{H}=\omega_{+}=0$ 


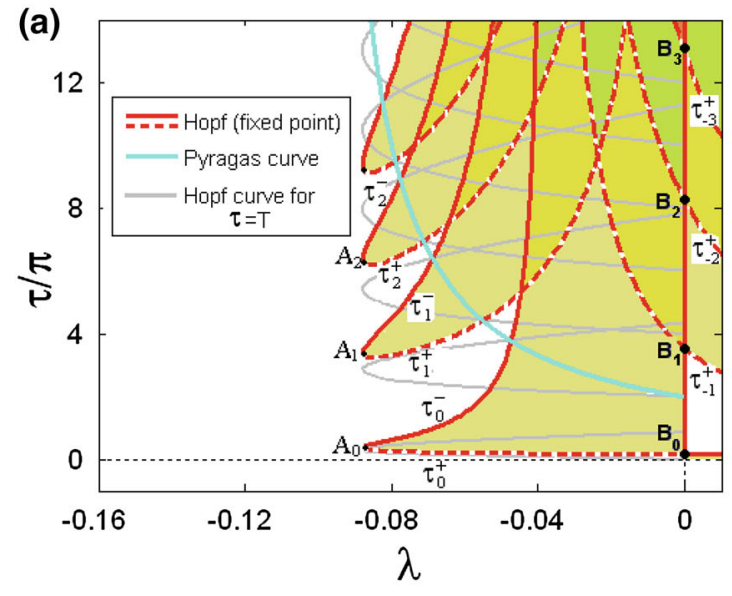

Fig. 1 (Color online) Two-dimensional bifurcation diagram of the fixed point in the $(\lambda, \tau)$-plane for $\omega_{0}=1, \gamma=-10, \beta=\pi / 4$, and $K=0.3$ : a destabilizing [dashed red (dark)] and stabilizing [solid red (dark)] Hopf curves $\tau_{n}^{ \pm}$for $\tau$-axis direction, Pyragas curve [solid cyan (light)], and Hopf curves of the conventional DFC (solid gray). Yellow (light gray) shading marks the domains of unstable fixed point,

gives $\bar{\eta}_{\lambda}=(1+i \gamma) /\left(1+K \tau e^{i \beta}\right)$, which yields

$\operatorname{Re} \bar{\eta}_{\lambda}=1+K \tau(\cos \beta+\gamma \sin \beta)$.

Thus, a change of the crossing directions of the critical Hopf eigenvalues for the $\lambda$-direction on the $\tau$ axis occurs at

$\tau^{*}=\frac{-1}{K(\cos \beta+\gamma \sin \beta)}$,

separating the $\tau$ axis as a Hopf curve into two part, i.e., the stabilization segment $\mathrm{H}^{-}$and destabilization segment $\mathrm{H}^{+}$. This is just the intersection point of the primary branch, $n=$ 0 , with $\tau$ axis given by Eq. (17). We note that it also coincides with the intersection point of the curve for the transcritical bifurcation of the UPO with the $\tau$ axis given by Eq. (29), as will be seen in Sect. 2.3.4 below. Therefore, the point $B_{0}$, i.e., $(\lambda, \tau)=\left(0, \tau^{*}\right)$, is a meeting point of three curves, i.e., two Hopf curves of the fixed point and a transcritical bifurcation curve of the UPO, at which each curve exhibits codimension-2 bifurcation.

\subsubsection{Numerical diagrams}

Our findings are summarized in Fig. 1 that shows a twodimensional bifurcation diagram of the fixed point in the $(\lambda, \tau)$-plane for $\gamma=-10, \beta=\pi / 4$, and $K=0.3$. The dashed and solid curves with red (dark) color indicate the stabilizing and destabilizing Hopf curves, $\tau_{n}^{+}$and $\tau_{n}^{-}$, for $\tau$-axis direction, respectively. The $\tau$ axis, i.e., $H=H^{+} \cup$ $H^{-}$, also forms a Hopf bifurcation curve. Codimension-2 bifurcations occur at the points $A_{n}$ and $B_{0}$. In Fig. 1b, we see that $B_{0}$ is a crossing point of three lines, two Hopf curves, $\tau_{0}^{+}$and $H=H^{+} \cup H^{-}$, and a transcritical curve (solid

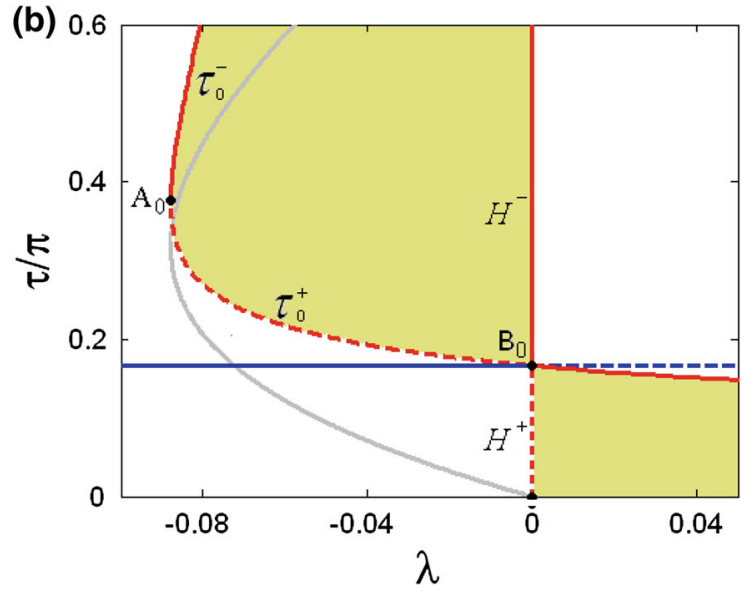

and the shading with the deeper color indicates the higher order of destabilization. b Same diagram enlarged close to the crossing point $B_{0}$ of three bifurcation curves. At the point $B_{0}$ two Hopf curves, $\tau_{0}^{+}$and $H=H^{+} \cup H^{-}$, and a transcritical curve of periodic orbits (solid blue line) exhibits codimension-2 bifurcation

blue) of the UPO (see also Fig. 2), while three codimension2 bifurcations occur at that point: stabilization directions are changed for the $\tau$-direction and $\lambda$-direction.

We note that the primary domain for destabilizing the fixed point, bounded by $\tau_{0}^{+}$and $\tau_{0}^{-}$, is much larger than that of the conventional DFC given by Eq. (6) and, moreover, contains not only the primary domain, but also most of the secondary one of conventional DFC. The domains of the destabilized fixed point, bounded by the Hopf curves, are marked by light gray shading (yellow online), which becomes darker in color with the increasing dimension of the unstable manifold.

The light gray curves denote Hopf curves with Pyragas delay, which was considered in [12] and [17]. The Pyragas curve given by Eq. (6) is seen in cyan color (light). When moving along the Pyragas curve, we clearly observe that these three curves cross each other in a point, reflecting that our control system is reduced to the Pyragas system as the delay time is chosen to be equal to the period of the UPO.

\subsubsection{Nonlinear feedback}

It is obvious that the control system (3) with the nonlinear feedback (5) yields the same characteristic equation of the fixed point as that of the linear case, Eq. (8), since $|z(t)|=$ $|z(t-\tau)|$ at the Hopf bifurcation. Therefore, the Hopf curves and the parameter region for destabilizing the fixed point are determined by the same equations as the linear feedback case.

\subsection{Stability analysis of the UPO}

As mentioned above, an unstable fixed point is neither a necessary nor a sufficient condition for stabilization of the UPO. 


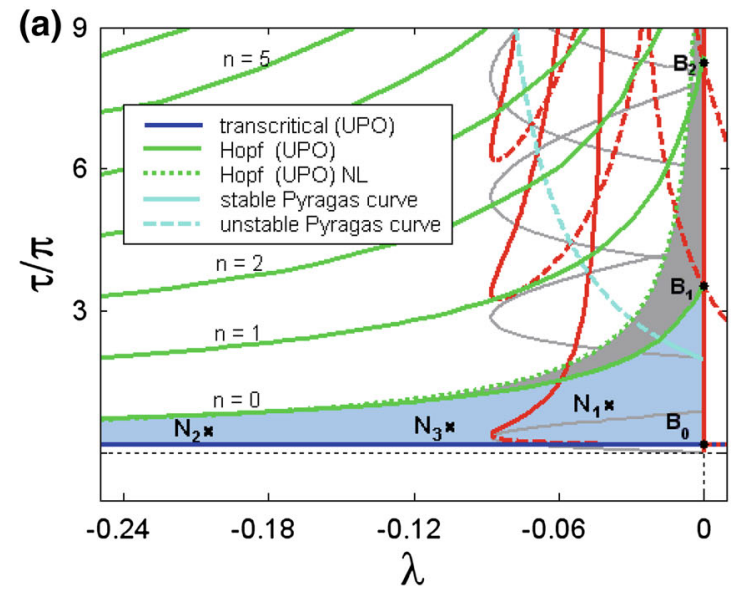

Fig. 2 (Color online) Same bifurcation diagram as in Fig. 1, including also the bifurcation curves for UPO: a transcritical bifurcation of the UPO [solid blue (dark)], Hopf bifurcation of the UPO for linear feedback [solid green (light)], Hopf bifurcation of the UPO for nonlinear feedback [dotted green (light)], stable and unstable Pyragas curve [solid and dashed cyan (light)]. Blue (light gray) shading marks the domains for stabilizing UPO using the linear feedback, and the dark gray shading corresponds to the enlarged part of the domain with the nonlinear feedback. Black crosses $N_{1}, N_{2}$ and $N_{3}$ denote points where numerical simulations for stabilizing UPO have been conducted using time series (see Fig. $4 \mathrm{a}-\mathrm{c}$ ). $N_{1}$ and $N_{2}$, which are lying sufficiently inside and out-

It affects only the global properties of the basin for the stabilization of the UPO: an overlapping region of parameters for simultaneously destabilizing the fixed point and stabilizing the UPO yields a global attractor basin for the domain inside the UPO, while the stabilization of the UPO alone guarantees its local stability.

\subsubsection{Floquet exponents}

Linear Feedback In polar coordinates $z=r e^{i \varphi}$, Eq. (3) with the linear feedback control (4) reads

$$
\begin{gathered}
\dot{r}=\left(\lambda+r^{2}\right) r+K\left[r ( t - \tau ) \operatorname { c o s } \left(\beta+\Omega_{0} \tau\right.\right. \\
+\varphi(t-\tau)-\varphi)-r \cos \beta] \\
\dot{\varphi}=\omega_{0}+\gamma r^{2}+K\left[\frac { r ( t - \tau ) } { r } \operatorname { s i n } \left(\beta+\Omega_{0} \tau\right.\right. \\
+\varphi(t-\tau)-\varphi)-\sin \beta]
\end{gathered}
$$

which obviously yields a noninvasive solution $r(t)=r_{0}$ and $\varphi(t)=\Omega_{0} t$ for any delay time $\tau$. Using the ansatz $r(t)=$ $r_{0}(1+\delta r(t)), \varphi(t)=\Omega_{0} t+\delta \varphi(t)$ and expanding Eqs. (26a, b) to linear order in the small deviations $\delta r$ and $\delta \varphi$, we obtain

$$
\begin{aligned}
\left(\begin{array}{c}
\dot{\delta} r \\
\dot{\delta} \varphi
\end{array}\right)= & \left(\begin{array}{ll}
-2 \lambda & 0 \\
-2 \gamma \lambda & 0
\end{array}\right)\left(\begin{array}{l}
\delta r \\
\delta \varphi
\end{array}\right) \\
& +K\left(\begin{array}{ll}
\cos \beta & -\sin \beta \\
\sin \beta & \cos \beta
\end{array}\right)\left(\begin{array}{l}
\delta r(t-\tau)-\delta r \\
\delta \varphi(t-\tau)-\delta \varphi
\end{array}\right),
\end{aligned}
$$

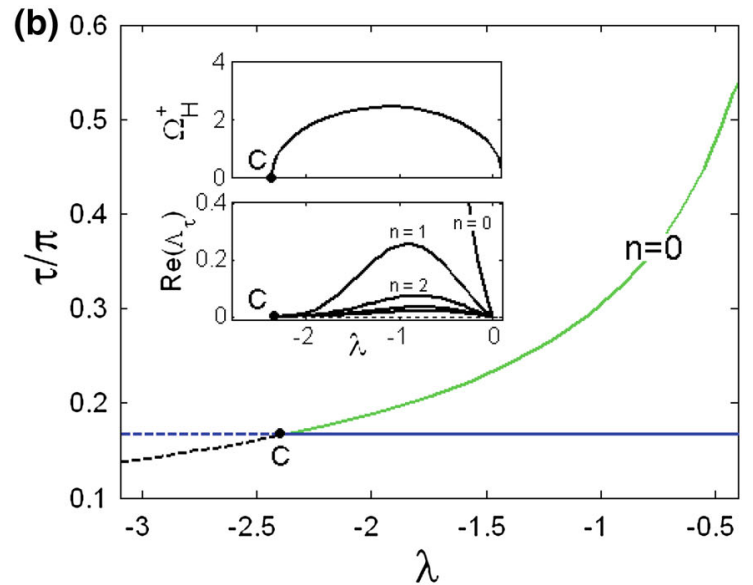

side the primary region for the unstable fixed point, correspond to points that represent the weakly and strongly unstable orbit, respectively, while $N_{3}$ indicates the point where the period of the UPO becomes infinite. b Enlarged part close to the crossing point $C$ of the transcritical and Hopf bifurcation curves of the UPO. Dotted blue (dark) line indicates the transcritical bifurcation curve for further destabilizing UPO for the $\tau$-axis direction. Dotted black (dark) curve corresponds to the spurious (unphysical) solution for Hopf bifurcation since the Hopf frequency takes imaginary values there, as shown in the insets. Parameters as in Fig. 1

Using the exponential ansatz $(\delta r(t), \delta \varphi(t)) \propto \exp (\Lambda t)$ gives a transcendental equation for the Floquet exponents $\Lambda$ as follows:

$\operatorname{det}\left[\begin{array}{ll}-2 \lambda+K\left(e^{-\Lambda \tau}-1\right) \cos \beta-\Lambda & -K\left(e^{-\Lambda \tau}-1\right) \sin \beta \\ -2 \gamma \lambda+K\left(e^{-\Lambda \tau}-1\right) \sin \beta & K\left(e^{-\Lambda \tau}-1\right) \cos \beta-\Lambda\end{array}\right]=0$,

that is,

$$
\begin{aligned}
0= & \chi_{1}(\Lambda) \equiv \Lambda^{2}+2[\lambda-K Q(\Lambda) \cos \beta] \Lambda \\
& +K^{2} Q^{2}(\Lambda)-2 \lambda K Q(\Lambda)(\cos \beta+\gamma \sin \beta)
\end{aligned}
$$

with $Q(\Lambda)=e^{-\Lambda \tau}-1$. Note that although the characteristic equation (27) has just the same form as that considered in Refs. $[12,17]$, now the time delay $\tau$ can take any positive value.

Nonlinear feedback For the nonlinear control (5), Eq. (3) can be written as follows:

$\dot{r}=\left(\lambda+r^{2}\right) r+K r\left[\cos \left(\beta+\Omega_{0} \tau+\varphi(t-\tau)-\varphi\right)-\cos \beta\right]$

$\dot{\varphi}=\omega+\gamma r^{2}+K\left[\sin \left(\beta+\Omega_{0} \tau+\varphi(t-\tau)-\varphi\right)-\sin \beta\right]$.

The variational equations around the UPO becomes

$$
\begin{aligned}
\left(\begin{array}{l}
\dot{\delta r} \\
\dot{\delta \varphi}
\end{array}\right)= & \left(\begin{array}{ll}
-2 \lambda & 0 \\
-2 \gamma \lambda & 0
\end{array}\right)\left(\begin{array}{l}
\delta r \\
\delta \varphi
\end{array}\right) \\
& +K\left(\begin{array}{ll}
0 & -\sin \beta \\
0 & \cos \beta
\end{array}\right)\left(\begin{array}{l}
\delta r(t-\tau)-\delta r \\
\delta \varphi(t-\tau)-\delta \varphi
\end{array}\right),
\end{aligned}
$$




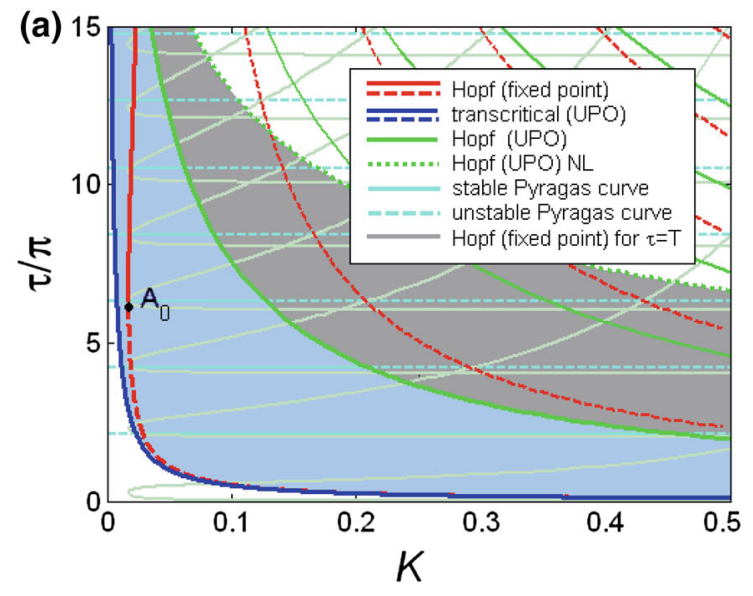

Fig. 3 (Color online) Two-dimensional bifurcation diagram of both the fixed point and, in pronounced form, UPO in the $(K, \tau)$-plane for $\gamma=-10, \beta=\pi / 4$, and $\lambda=-0.005$ : a transcritical bifurcation of the UPO [solid blue (dark)], Hopf bifurcation of the UPO for linear feedback [solid green (light)], Hopf bifurcation of the UPO for nonlinear feedback [dotted green (light)], stable [solid cyan (light)] and unstable [dashed cyan (light)] Pyragas curve. Blue (light gray) shading marks the domains for stabilizing UPO using the linear feedback, and the gray shading corresponds to the enlarged part of the domain with the nonlinear feedback. b Enlarged part close to the crossing point $C$ of the

which yields

$\operatorname{det}\left[\begin{array}{ll}-2 \lambda-\Lambda & -K\left(e^{-\Lambda \tau}-1\right) \sin \beta \\ -2 \gamma \lambda & K\left(e^{-\Lambda \tau}-1\right) \cos \beta-\Lambda\end{array}\right]=0$,

i.e., equation for the Floquet exponents $\Lambda$ for the nonlinear feedback control (5)

$$
\begin{aligned}
0= & \chi_{2}(\Lambda) \equiv \Lambda^{2}+[2 \lambda-K Q(\Lambda) \cos \beta] \Lambda \\
& -2 \lambda K Q(\Lambda)(\cos \beta+\gamma \sin \beta) .
\end{aligned}
$$

We note that $\chi_{2}(\Lambda)$ contains only the first order terms of the transcendental function $Q(\Lambda)$, which allows to perform a thorough bifurcation analysis of the UPO. It also yields a larger parameter region for the stabilization of the target UPO than the case of $\chi_{1}(\Lambda)$ that includes not only the first order, but also the second order, as seen below.

\subsubsection{Transcritical bifurcation of the UPO}

Point of Transcritical Bifurcation Note that $\Lambda=0$ is always a solution of Eq. (27), which corresponds to the Goldstone mode of the periodic orbit, i.e., the trivial Floquet mode with Floquet exponent zero. A second, but nontrivial vanishing eigenvalue is generated when the transcritical bifurcation of periodic orbits occurs. If we confine $\Lambda$ within real eigenvalues, then the condition for a transcritical bifurcation in the control system is provided by a double zero of

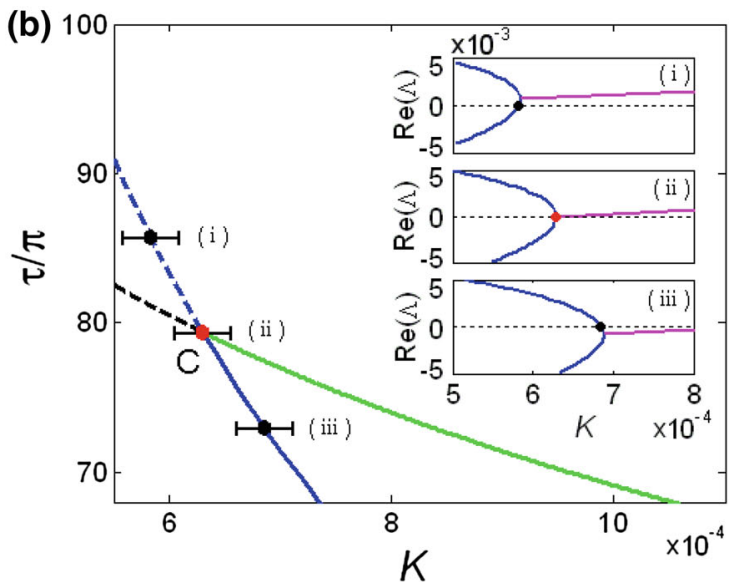

transcritical and Hopf bifurcation curves of the UPO. Dotted blue (dark) line indicates the transcritical bifurcation curve for further destabilizing UPO for the $\tau$-axis direction. Dotted black (dark) curve corresponds to the spurious (unphysical) solution for Hopf bifurcation. Insets (i), (ii) and (iii) display the real part of Floquet exponents in dependence on the control amplitude $K$ for the delay time given by $\tau^{*}+20, \tau^{*}$ and $\tau^{*}-20$ with $\tau^{*}=249.4$, respectively. The blue (dark) and magenta (light) lines in the insets correspond to the transcritical mode (with zero imaginary part) and Hopf mode (with non-zero imaginary part), respectively

$$
\begin{aligned}
& 0=\chi_{1}(\Lambda ; K, \tau, \lambda), \text { i.e., } \\
& 0=\chi_{1}(0 ; K, \tau, \lambda), \\
& 0=\frac{\partial}{\partial \Lambda} \chi_{1}(0 ; K, \tau, \lambda) .
\end{aligned}
$$

Thus, the point of transcritical bifurcation is simply given as

$$
\tau^{T r}=-\frac{1}{K(\cos \beta+\gamma \sin \beta)},
$$

which is independent of the parameter $\lambda$. Note that this coincides with the intersection point of the primary Hopf branch of the fixed point with $\tau$ axis given by Eq. (17) and with the boundary value of $H=H^{+} \cup H^{-}$given by Eq. (25).

The approach of $\tau_{0}^{+}$to $\tau^{T r}$ as $\lambda \rightarrow 0$ may be observed also from Fig. 3a, where two curves, i.e., the primary Hopf branch of the fixed point (red color) and the transcritical curve (blue color), are lying close to each other for small parameter $\lambda=-0.005$. The nonlinear feedback control with Eq. (5) also gives the same expression for the transcritical bifurcation as Eq. (29).

Turning Point of the Stability Direction We calculate the analytical conditions for the change of the stabilization direction that occurs on the transcritical curve, the codimension-2 bifurcation. Eliminating the Goldstone mode, a transcendental function $G_{1}(\Lambda)=\chi_{1}(\Lambda) / \Lambda$ is considered instead of 
$\chi_{1}(\Lambda)$. Taking into account the derivatives

$\left.\frac{\partial G_{1}}{\partial \tau}\right|_{\Lambda=0}=2 \lambda K(\cos \beta+\gamma \sin \beta)$

and

$$
\begin{aligned}
& \left.\frac{\partial G_{1}}{\partial \Lambda}\right|_{\Lambda=0} \\
& =\lim _{\Lambda \rightarrow 0}\left(1-2 K Q^{\prime} \cos \beta\right. \\
& \left.\quad+\frac{2 K^{2} Q Q^{\prime} \Lambda-2 \lambda K Q^{\prime} \Lambda(\cos \beta+\gamma \sin \beta)-K^{2} Q^{2}+2 \lambda K Q(\cos \beta+\gamma \sin \beta)}{\Lambda^{2}}\right) \\
& =\lim _{\Lambda \rightarrow 0}\left\{1+2 K \tau \cos \beta+K^{2}\left(Q^{\prime 2}+Q Q^{\prime \prime}\right)-\lambda K Q^{\prime \prime}(\cos \beta+\gamma \sin \beta)\right\} \\
& =1+2 K \tau \cos \beta+K^{2} \tau^{2}-\lambda K \tau^{2}(\cos \beta+\gamma \sin \beta),
\end{aligned}
$$

we obtain

$$
\begin{aligned}
& \left.\frac{\partial \Lambda}{\partial \tau}\right|_{\Lambda=0}=-\left.\frac{\frac{\partial G_{1}}{\partial \tau}}{\frac{\partial G_{1}}{\partial \Lambda}}\right|_{\Lambda=0} \\
& =\frac{-2 \lambda K(\cos \beta+\gamma \sin \beta)}{1+2 K \tau \cos \beta+K^{2} \tau^{2}-\lambda K \tau^{2}(\cos \beta+\gamma \sin \beta)} .
\end{aligned}
$$

It is obvious that the sign of $\Lambda_{\tau}$ is always positive for $\lambda>0$, since $\lambda K(\cos \beta+\gamma \sin \beta)<0$ and $1+2 K \tau \cos \beta+$ $K^{2} \tau^{2}>0$. On the other hand, for the case of $\lambda<0$, Eq. (30) exchanges its sign when its denominator becomes zero, i.e., at

$$
\begin{aligned}
\lambda=\lambda^{*} & \equiv \frac{-\left(1+\gamma^{2}\right) \sin ^{2} \beta}{\tau(\cos \beta+\gamma \sin \beta)^{2}}, \\
& =\frac{K\left(1+\gamma^{2}\right) \sin ^{2} \beta}{\cos \beta+\gamma \sin \beta},
\end{aligned}
$$

where the condition for the transcritical bifurcation, i.e., $1+K \tau(\cos \beta+\gamma \sin \beta)=0$, was used.

Therefore, the transcritical curve in the intervals $\lambda^{*}<$ $\lambda<0$ and $\lambda<\lambda^{*}$ represents the boundaries for stabilization and destabilization of the UPO for the positive $\tau$-direction, respectively. The transition point $C$ characterized by $\lambda^{*}$ can be expressed in the $(\lambda, \tau)$-plane and the $(K, \tau)$-plane as

$$
\left(\lambda^{*}, \tau^{*}\right)=\left(\frac{K\left(1+\gamma^{2}\right) \sin ^{2} \beta}{\cos \beta+\gamma \sin \beta}, \frac{-1}{K(\cos \beta+\gamma \sin \beta)}\right),
$$

and

$$
\left(K^{*}, \tau^{*}\right)=\left(\frac{\lambda(\cos \beta+\gamma \sin \beta)}{\left(1+\gamma^{2}\right) \sin ^{2} \beta}, \frac{-\left(1+\gamma^{2}\right) \sin ^{2} \beta}{\lambda(\cos \beta+\gamma \sin \beta)^{2}}\right),
$$

respectively. Note that the derivative $\left.\frac{\partial \Lambda}{\partial \tau}\right|_{\Lambda=0}$ becomes infinite at the codimension-2 bifurcation point $(K, \tau)=$ $\left(K^{*}, \tau^{*}\right)$, while it takes positive and negative values for $\tau$ above and below the transcritical curve, respectively, as shown in the insets of Fig. $3 \mathrm{~b}$.
The condition for stability and instability for $\lambda^{*}<\lambda<0$ can be written as

$\tau \begin{cases}>\tau^{T r}, & \text { transcritical stable, } \\ <\tau^{T r}, & \text { transcritical unstable. }\end{cases}$

Similarly, for the nonlinear feedback control with Eq. (5), we obtain the following relations:

$\left.\frac{\partial G_{2}}{\partial \tau}\right|_{\Lambda=0}=2 \lambda K(\cos \beta+\gamma \sin \beta)$,

and

$\left.\frac{\partial G_{2}}{\partial \Lambda}\right|_{\Lambda=0}=1+K \tau \cos \beta-\lambda K \tau^{2}(\cos \beta+\gamma \sin \beta)$,

which yields

$\left.\frac{\partial \Lambda}{\partial \tau}\right|_{\Lambda=0} \begin{cases}>0 & \text { for } 0<\lambda, \\ <0 & \text { for } \lambda^{*}<\lambda<0, \\ >0 & \text { for } \lambda<\lambda^{*},\end{cases}$

where $G_{2}(\Lambda)=\chi_{2}(\Lambda) / \Lambda$ and $\lambda^{*}=\frac{-\gamma \sin \beta}{\tau(\cos \beta+\gamma \sin \beta)}$. The boundary point for the transition can be written in the $(\lambda, \tau)$ plane and the $(K, \tau)$-plane by

$\left(\lambda^{*}, \tau^{*}\right)=\left(\gamma K \sin \beta, \frac{-1}{K(\cos \beta+\gamma \sin \beta)}\right)$,

and

$\left(K^{*}, \tau^{*}\right)=\left(\frac{\lambda}{\gamma \sin \beta}, \frac{-\gamma \sin \beta}{\lambda(\cos \beta+\gamma \sin \beta)}\right)$,

respectively. 


\subsubsection{Hopf bifurcation of the UPO}

The transcritical stable region could be cut off by an appearance of torus bifurcation, i.e., Hopf bifurcation of the UPO.

Linear Feedback One can find the Hopf bifurcation of the UPO (in a semi-analytic way) by inserting $\Lambda=i \Omega_{H}$ into Eq. (27) and separating the equation into real and imaginary parts as follows:

Real: $A_{1} K^{2}+B_{1} K+C_{1}=0$,

Imag: $A_{2} K^{2}+B_{2} K+C_{2}=0$,

where

$$
\begin{aligned}
& A_{1}=2 \cos \left(\Omega_{H} \tau\right)\left[\cos \left(\Omega_{H} \tau\right)-1\right], \\
& A_{2}=2 \sin \left(\Omega_{H} \tau\right)\left[1-\cos \left(\Omega_{H} \tau\right)\right], \\
& B_{1}=2 \delta\left[1-\cos \left(\Omega_{H} \tau\right)\right]-2 \Omega_{H} \cos \beta \sin \left(\Omega_{H} \tau\right), \\
& B_{2}=2 \Omega_{H} \cos \beta\left[1-\cos \left(\Omega_{H} \tau\right)\right]+2 \delta \sin \left(\Omega_{H} \tau\right), \\
& C_{1}=-\Omega_{H}^{2}, C_{2}=2 \lambda \Omega_{H}, \delta=\lambda(\cos \beta+\gamma \sin \beta) .
\end{aligned}
$$

Multiplying Eq. (38a) with $\sin \Omega_{H} \tau$ and Eq. (38b) with $\cos \Omega_{H} \tau$ leads to

$$
\begin{aligned}
& \left(2 K \delta-\Omega_{H}^{2}\right) \sin \left(\Omega_{H} \tau\right) \\
& \quad+2 \Omega_{H}(K \cos \beta+\lambda) \cos \left(\Omega_{H} \tau\right)=2 K \Omega_{H} \cos \beta \\
& -2 \Omega_{H}(K \cos \beta+\lambda) \sin \left(\Omega_{H} \tau\right)+\left(2 K \delta-\Omega_{H}^{2}\right. \\
& \left.+2 K^{2}\right) \cos \left(\Omega_{H} \tau\right)=2 K(\delta+K) .
\end{aligned}
$$

Solving Eqs. (39a, b) with respect to $\sin \left(\Omega_{H} \tau\right)$ and $\cos \left(\Omega_{H} \tau\right)$ yields

$$
\sin \left(\Omega_{H} \tau\right)=H_{S}\left(\Omega_{H}, \lambda, K\right),
$$

$\cos \left(\Omega_{H} \tau\right)=H_{c}\left(\Omega_{H}, \lambda, K\right)$

where the right-hand sides are given by

$$
\begin{aligned}
& H_{S}\left(\Omega_{H}, \lambda, K\right) \\
& =\frac{2 K \Omega_{H}\left[\left(2 K \delta-\Omega_{H}^{2}+2 K^{2}\right) \cos \beta-2(\delta+K)(K \cos \beta+\lambda)\right]}{\left(2 K \Omega_{H} \cos \beta+2 \lambda \Omega_{H}\right)^{2}+\left(2 K \delta-\Omega_{H}^{2}\right)\left(2 K \delta-\Omega_{H}^{2}+2 K^{2}\right)}, \\
& H_{C}\left(\Omega_{H}, \lambda, K\right) \\
& =\frac{2 K \Omega_{H}\left(2 K \Omega_{H} \cos \beta+2 \lambda \Omega_{H}\right) \cos \beta+2 K(\delta+K)\left(2 K \delta-\Omega_{H}^{2}\right)}{\left(2 K \Omega_{H} \cos \beta+2 \lambda \Omega_{H}\right)^{2}+\left(2 K \delta-\Omega_{H}^{2}\right)\left(2 K \delta-\Omega_{H}^{2}+2 K^{2}\right)},
\end{aligned}
$$

Using the trigonometric identity $\cos ^{2}\left(\Omega_{H} \tau\right)+\sin ^{2}\left(\Omega_{H} \tau\right)=$ 1 , we obtain the 8 th degree polynomial equation

$$
0=\Omega_{H}^{8}+a_{2}(\lambda, K) \Omega_{H}^{6}+a_{1}(\lambda, K) \Omega_{H}^{4}+a_{0}(\lambda, K) \Omega_{H}^{2},
$$

where we used the abbreviations

$$
\begin{aligned}
a_{2}= & 8 \lambda^{2}+8 K(\cos \beta-\gamma \sin \beta) \lambda-4 K^{2} \sin ^{2} \beta, \\
a_{1}= & 16 \lambda^{4}+32 K(\cos \beta-\gamma \sin \beta) \lambda^{3} \\
& +4 K^{2}\left(5 \gamma^{2} \sin ^{2} \beta-6 \gamma \cos \beta \sin \beta-4+9 \cos ^{2} \beta\right) \lambda^{2} \\
& -16 K^{3} \sin ^{2} \beta(\cos \beta-\gamma \sin \beta) \lambda, \\
a_{0}= & 16 \lambda^{2} K^{2}\left[(\cos \beta+\gamma \sin \beta)^{2} \lambda^{2}+K(\cos \beta+\gamma \sin \beta)^{2}\right. \\
& \left.\times(\cos \beta-\gamma \sin \beta) \lambda-K^{2}\left(1+\gamma^{2}\right) \sin ^{2} \beta\right] \\
= & 16 \lambda^{2} K^{2}[(\cos \beta+\gamma \sin \beta) \lambda+K][(\cos \beta+\gamma \sin \beta) \lambda \\
& \left.-K\left(1+\gamma^{2}\right) \sin ^{2} \beta\right] .
\end{aligned}
$$

Two roots of Eq. (41), $\Omega_{H 1}=0$ and $\Omega_{H 2}=0$, correspond to the Goldstone mode and the transcritical mode, respectively. The Hopf frequencies are determined by $\Omega_{H}=\Omega_{H}^{ \pm} \equiv$ $\pm \sqrt{\Gamma}$, where $\Gamma>0$ is given by the roots of the following 3rd order polynomial

$\Gamma^{3}+a_{2} \Gamma^{2}+a_{1} \Gamma+a_{0}=0$.

Note that, without loss of generality, it is enough to consider only one, e.g., $\Omega_{H}^{+}=+\sqrt{\Gamma}$, of the two frequencies $\pm \sqrt{\Gamma}$ since Eqs. (38a, b) is invariant with respective to the transformation $\Omega_{H} \rightarrow-\Omega_{H}$. Then, we obtain an analytical expression for the Hopf curve of the UPO from Eq. (40a):

$$
\begin{aligned}
\tau_{n}^{H} & =\frac{+\arccos \left(H_{c}\right)+2 n \pi}{\Omega_{H}^{+}} \text {if } H_{s}>0, \\
\tau_{n}^{H} & =\frac{-\arccos \left(H_{c}\right)+2(n+1) \pi}{\Omega_{H}^{+}} \text {if } H_{s}<0,
\end{aligned}
$$

where $n=0,1,2, \ldots$ In Eq. (43b), the integer $n$ was renumbered by $n+1$ such that the delay times take the positive values from the primary branch, $\tau_{0}^{H}$.

The crossing point of Hopf curves of the UPO along the $\tau$ axis is given by

$\tau_{n *}^{H}=\frac{-\beta+(n+1) \pi}{K \sin \beta}$ for $n=0,1,2, \ldots$,

since $\Omega_{H}^{+}=2 K \sin \beta, H_{c}=\cos (2 \beta)$ and $H_{s}=-\sin$ $(2 \beta)<0$ for $\lambda=0$. These coincide with the crossing points of Hopf curves of the fixed point along the $\tau$ axis, i.e., , $B_{1}, B_{2}, \ldots$, given by Eq. (18b), which can be seen in Fig. 2a.

In order to evaluate the crossing direction of the Floquet exponent $\Lambda$ through the Hopf curves of the UPO for the $\tau$-direction, we calculate numerically the following derivative: 
$\left.\operatorname{Re}\left(\frac{\partial \Lambda}{\partial \tau}\right)\right|_{\tau=\tau_{n}^{H}}$

Note that the Hopf frequency vanishes again at the points where the constant term $a_{0}$ of Eq. (42) becomes zero, i.e.,

$\lambda_{1}=\frac{K\left(1+\gamma^{2}\right) \sin ^{2} \beta}{\cos \beta+\gamma \sin \beta}<0, \quad$ and $\lambda_{2}=\frac{-K}{\cos \beta+\gamma \sin \beta}>0$,

of which the former coincides with the $\lambda$-coordinate of the point $C$ given by Eq. (31b). The Hopf bifurcation of the UPO occurs only for $\lambda \in\left(\lambda_{1}, 0\right)$ and, otherwise, there exist unphysical solutions. Numerical calculations show that the derivative is positive for any $n$ in the interval $\lambda \in\left(\lambda_{1}, 0\right)$, as shown in the inset of Fig. 2b. Thus, whenever crossing through the Hopf curves for the positive $\tau$-direction, only destabilization of the UPO occurs for any $n$, while there exists no point of codimension-2 bifurcation on the curves.

Since $\tau_{0}^{H}<\tau_{1}^{H}<\tau_{2}^{H} \cdots$, only the primary Hopf curve with $n=0$ gives a contribution to the boundary of the control domain for stabilization of the UPO, and we obtain the necessary and sufficient condition for stabilizing the UPO as follows:

$\tau^{T r}<\tau<\tau_{0}^{H}$

Nonlinear Feedback In case of the nonlinear feedback control, it is possible to thoroughly perform the analytical study. With abbreviations $a=-2 \lambda, b=K \cos \beta$ and $c=-2 \lambda K(\cos \beta+\gamma \sin \beta)=-2 K \delta$, Eq. (28) is rewritten as

$\Lambda^{2}-\left[a+b\left(e^{-\Lambda \tau}-1\right)\right] \Lambda+c\left(e^{-\Lambda \tau}-1\right)=0$.

A Hopf bifurcation of the UPO occurs for $\Lambda=i \Omega_{H}$, which gives

$\Omega_{H}^{2}+c=-b \Omega_{H} \sin \left(\Omega_{H} \tau\right)+c \cos \left(\Omega_{H} \tau\right)$,

$(a-b) \Omega_{H}=-c \sin \left(\Omega_{H} \tau\right)-b \Omega_{H} \cos \left(\Omega_{H} \tau\right)$.

Using the trigonometric identity $\left[\cos \left(\beta-\Omega_{H} \tau\right)\right]^{2}+[\sin$ $\left.\left(\beta-\Omega_{H} \tau\right)\right]^{2}=1$, the Hopf frequency is determined by

$\Omega_{H}^{4}+\left[2 c+(a-b)^{2}-b^{2}\right] \Omega_{H}^{2}=0$,

which yields the solution

$\Omega_{H 1,2}=0$,

$\Omega_{H 3,4}= \pm \bar{\Omega}$

where

$$
\begin{aligned}
\bar{\Omega} & =\sqrt{2 a b-a^{2}-2 c} \\
& =2 \sqrt{K \lambda \gamma \sin \beta-\lambda^{2}} .
\end{aligned}
$$

The vanishing frequencies $\Omega_{H 1}=0$ and $\Omega_{H_{2}}=0$ correspond to the Goldstone mode and the transcritical mode, respectively. Note that the non-vanishing Hopf frequency is defined only for

$K \gamma \sin \beta<\lambda<0$,

or, equivalently,

$K>\frac{\lambda}{\gamma \sin \beta}$

In particular, the point for the vanishing Hopf frequency, $\lambda=K \gamma \sin \beta$, or $K=\lambda /(\gamma \sin \beta)$, coincides with the codimension- 2 bifurcation point on the transcritical curve of the UPO given by Eqs. (36) and (37).

Without loss of generality, it is again enough to consider only one of the frequencies $\Omega_{H}= \pm \bar{\Omega}$. Then, for $\Omega_{H}=+\bar{\Omega}$, Eqs. (46a, b) can be rewritten as

$\cos (\bar{\Omega} \tau+\alpha)=\frac{\bar{\Omega}^{2}+c}{\sqrt{b^{2} \bar{\Omega}^{2}+c^{2}}}$,
$\sin (\bar{\Omega} \tau+\alpha)=\frac{(b-a) \bar{\Omega}}{\sqrt{b^{2} \bar{\Omega}^{2}+c^{2}}}$,

where $\cos \alpha=c / \sqrt{b^{2} \bar{\Omega}^{2}+c^{2}}(<0)$ and $\sin \alpha=$ $b \bar{\Omega} / \sqrt{b^{2} \bar{\Omega}^{2}+c^{2}}(>0)$, and thus $\frac{\pi}{2}<\alpha=\arccos \frac{c}{\sqrt{b^{2} \bar{\Omega}^{2}+c^{2}}}$ $<\pi$. Therefore, we obtain analytical expression for the occurrence of the Hopf bifurcation of the UPO

$\bar{\Omega} \tau+\alpha=+\arccos \left(\frac{\bar{\Omega}^{2}+c}{\sqrt{b^{2} \bar{\Omega}^{2}+c^{2}}}\right)+2 n \pi \quad$ if $b>a$,

$\bar{\Omega} \tau+\alpha=-\arccos \left(\frac{\bar{\Omega}^{2}+c}{\sqrt{b^{2} \bar{\Omega}^{2}+c^{2}}}\right)+2 n \pi \quad$ if $b<a$,

where $n=0, \pm 1, \pm 2, \ldots$. Then, we obtain the following analytical expressions for the Hopf curve of the UPO for the nonlinear feedback control:

$$
\begin{aligned}
\tau_{n}^{H}= & \frac{1}{\bar{\Omega}}\left[+\arccos \left(\frac{\bar{\Omega}^{2}+c}{\sqrt{b^{2} \bar{\Omega}^{2}+c^{2}}}\right)-\arccos \left(\frac{c}{\sqrt{b^{2} \bar{\Omega}^{2}+c^{2}}}\right)\right. \\
& +2(n+1) \pi] \text { for } K>-2 \lambda / \cos \beta, \\
\tau_{n}^{H}= & \frac{1}{\bar{\Omega}}\left[-\arccos \left(\frac{\bar{\Omega}^{2}+c}{\sqrt{b^{2} \bar{\Omega}^{2}+c^{2}}}\right)-\arccos \left(\frac{c}{\sqrt{b^{2} \bar{\Omega}^{2}+c^{2}}}\right)\right. \\
& +2(n+1) \pi] \text { for } K<-2 \lambda / \cos \beta,
\end{aligned}
$$

where $n=0,1,2, \ldots$ In Eqs. (48a, b), the integer $n$ was renumbered by $n+1$ such that the delay times including the primary branch, $\tau_{0}^{H}$, take positive values. 
In order to evaluate the crossing direction of the Floquet exponent $\Lambda$ through the Hopf curves for the $\tau$-direction, we calculate the following derivatives:

$$
\frac{\partial \Lambda}{\partial \tau}=-\frac{\frac{\partial \chi_{2}}{\partial \tau}}{\frac{\partial \chi_{2}}{\partial \Lambda}}=\frac{(c-b \Lambda) e^{-\Lambda \tau}}{2 \Lambda-\left[a+b\left(e^{-\Lambda \tau-1}\right)\right]+\tau(b \Lambda-c) e^{-\Lambda \tau}}
$$

and

$$
\begin{aligned}
\left.\frac{\partial \Lambda}{\partial \tau}\right|_{\Lambda=i \tilde{\Omega}}= & \frac{1}{D}\left[b \tilde{\Omega}^{2} \cos \tilde{\Omega} \tau+c \tilde{\Omega} \sin \tilde{\Omega} \tau\right. \\
& \left.-i\left(b \tilde{\Omega}^{2} \sin \tilde{\Omega} \tau-c \tilde{\Omega} \cos \tilde{\Omega} \tau\right)\right],
\end{aligned}
$$

where $D=-a+b-b \cos \tilde{\Omega} \tau+b \tilde{\Omega} \tau \sin \tilde{\Omega} \tau-c \tau \cos \tilde{\Omega} \tau$ $+i(2 \tilde{\Omega}+b \sin \tilde{\Omega} \tau+b \tilde{\Omega} \tau \cos \tilde{\Omega} \tau+c \tau \sin \tilde{\Omega} \tau)$. Then, we obtain the following simple result:

$$
\begin{aligned}
\left.\operatorname{Re}\left(\frac{\partial \Lambda}{\partial \tau}\right)\right|_{\Lambda=i \tilde{\Omega}}= & \frac{1}{|D|^{2}}[\tilde{\Omega}(b-a)(b \tilde{\Omega} \cos \tilde{\Omega} \tau+c \sin \tilde{\Omega} \tau) \\
& \left.-2 \tilde{\Omega}^{2}(b \tilde{\Omega} \sin \tilde{\Omega} \tau-c \cos \tilde{\Omega} \tau)-b^{2} \tilde{\Omega}^{2}\right] \\
= & \frac{1}{|D|^{2}}\left(2 a b-a^{2}-2 c\right) \tilde{\Omega}^{2}=\frac{\tilde{\Omega}^{4}}{|D|^{2}}>0 .
\end{aligned}
$$

Thus, whenever crossing through the Hopf curves for the positive $\tau$-direction, only destabilization of the UPO occurs for any $n$, while there exists no point of codimension- 2 bifurcation on the curves. Since $\tau_{0}^{H}<\tau_{1}^{H}<\tau_{2}^{H} \cdots$, only the primary Hopf curve with $n=0$ makes a contribution to the boundary of the control domain for stabilization of the UPO, and we obtain the necessary and sufficient condition for stabilizing the UPO as follows: $\tau^{T r}<\tau<\tau_{0}^{H}$.

\subsubsection{Intersection of the transcritical and Hopf curves}

There exists a region of $\tau$ for stabilizing the UPO in Eq. (45) only in the case of $\tau^{T r}<\tau_{0}^{H}$. The intersection of the two curves occurs at the point in parameter space where Eqs. (27) and (28) have a triple real root.

For linear feedback, this point is determined by

$$
\begin{aligned}
& \chi_{1}(0)=0, \\
& \chi_{1}^{\prime}(0)=0, \\
& \chi_{1}^{\prime \prime}(0)=0,
\end{aligned}
$$

which yield the position of the intersection point $C$ as

$$
\left(K_{C}, \tau_{C}\right)=\left(\frac{\lambda(\cos \beta+\gamma \sin \beta)}{\left(1+\gamma^{2}\right) \sin ^{2} \beta}, \frac{-\left(1+\gamma^{2}\right) \sin ^{2} \beta}{\lambda(\cos \beta+\gamma \sin \beta)^{2}}\right) .
$$

For the nonlinear feedback, the condition for Eq. (28) to have the triple real root yields

$$
K_{C}=\frac{\lambda}{\gamma \sin \beta} \quad \text { and } \quad \tau_{C}=\frac{-\gamma \sin \beta}{\lambda(\cos \beta+\gamma \sin \beta)} .
$$

The points satisfying Eqs. (49) and (50) represent just the same points as Eqs. (32)-(33) and Eqs. (36)-(37), respectively.

Thus, the intersection of the transcritical curve and Hopf curve occurs at a point that coincides with the point $C$ of the codimension-2 bifurcation on the transcritical curve (see Figs. 2, 3).

\subsection{Numerical bifurcation diagrams}

Figure 2 displays a two-dimensional bifurcation diagram of the UPO as well as the fixed point in the $(\lambda, \tau)$-plane. The parameters are the same as in Fig. 1. The diagram contains a transcritical bifurcation line of the UPO [solid blue (dark)], a family of Hopf bifurcation lines of the UPO for linear feedback [solid green (light)], a Hopf bifurcation of the UPO for nonlinear feedback [dotted green (light)], and stable and unstable Pyragas curves [solid and cyan (light)]. Blue (light gray) shading marks the domains of stabilization of the target orbit using the linear feedback, of which boundaries are formed by Eqs. (29) and (43a, 43b) with $n=0$. The gray shading with the boundary equation (48) corresponds to the augmented part of the successful control domain with the nonlinear feedback control system, Eqs. (3) and (5), which shows the advantages of the nonlinear control scheme over the linear one. Black crosses $N_{1}, N_{2}$, and $N_{3}$ denote points where the numerical simulations for stabilization of the target orbit have been conducted using time series (see Fig. $4 a-c$ ).

Figure 3 depicts a two-dimensional bifurcation diagram of both the fixed point and, in pronounced form, the UPO in the $(K, \tau)$-plane for $\gamma=-10, \beta=\pi / 4$, and $\lambda=-0.005$ : In Fig. 3a, a transcritical bifurcation of the UPO [solid blue (dark)], Hopf bifurcation of the UPO for linear feedback [solid green (light)], a Hopf bifurcation of the UPO for nonlinear feedback [dotted green (light)], stable [solid cyan (light)] and unstable [dashed cyan (light)] Pyragas curves can be seen. Blue (light gray) shading marks the domains of stabilization of the target orbit using the linear feedback, and the gray shading corresponds to the enlarged part of the domain with the nonlinear feedback. Figure $3 b$ is enlarged part close to the crossing point $C$ of the transcritical and Hopf bifurcation curves of the UPO, determined by $(K, \tau)=\left(K^{*}, \tau^{*}\right)$. The dotted blue (dark) line indicates the transcritical bifurcation curve for further destabilizing the orbit for the $\tau$-axis direction and the dotted black (dark) curve corresponds to the spurious (unphysical) solution for Hopf bifurcation. Insets (i), (ii), and (iii) of Fig. 3b display the real part of the leading Floquet exponents in dependence on the control amplitude $K$ for the delay time given by $\tau^{*}+20, \tau^{*}$ and $\tau^{*}-20$ with $\tau^{*}=249.4$, respectively. The blue (dark) and magenta (light) 

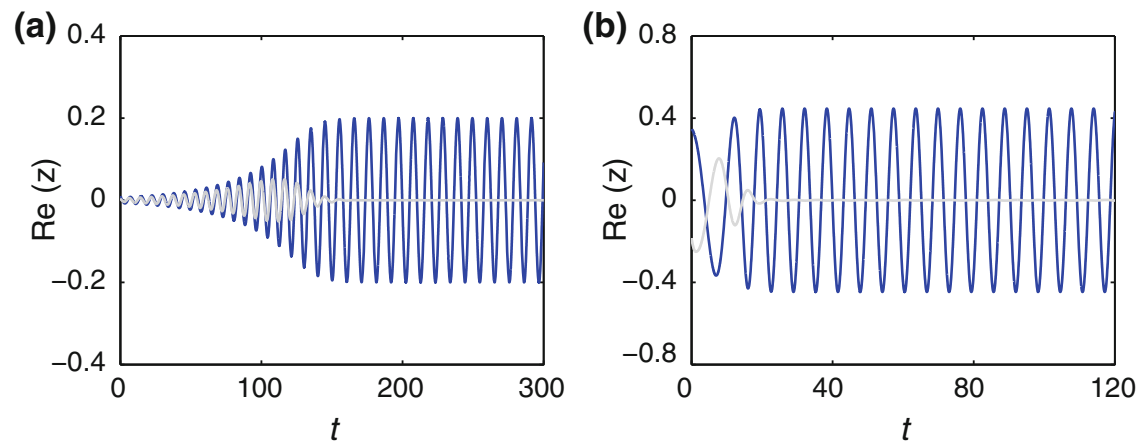

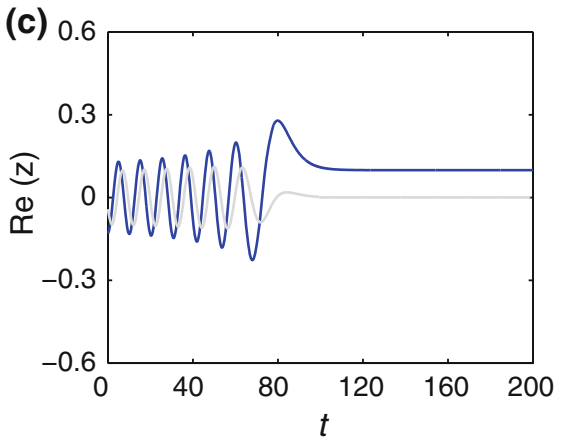

Fig. 4 (Color online) Time series of $\operatorname{Re}(z)$ for three different parameter sets $(\lambda, \tau)$ : a-c correspond to the black crosses $N_{1}$ with $(\lambda, \tau)=$ $(-0.04,0.9 \pi), N_{2}$ with $(\lambda, \tau)=(-0.2,0.4 \pi)$ and $N_{3}$ with $(\lambda, \tau)=$ $(-0.1,0.4 \pi)$ of Fig. 2, respectively. The point $N_{1}$ is representative for the case of simultaneous destabilization of the fixed point and stabilization of the target orbit, while $N_{2}$ stands for periodic orbit stabilization alone. a The system might start arbitrarily close to $z=0$, which means that the basin of attraction for stabilizing the UPO includes the whole

lines in the insets correspond to the transcritical mode (with zero imaginary part) and Hopf mode (with non-zero imaginary part), respectively. Note that the gradient of the transcritical mode curve at $\Lambda=0$ varies from positive to infinity, and to negative in the insets (i), (ii), and (iii), respectively.

Time series obtained by numerical calculations are displayed in Fig. 4. Parameter sets of Fig. 4a-c correspond to the black crosses $N_{1}, N_{2}$, and $N_{3}$ in Fig. 2, and are given by $(\lambda, \tau)=(-0.04,0.9 \pi),(\lambda, \tau)=(-0.2,0.4 \pi)$ and $(\lambda, \tau)=(-0.1,0.9 \pi)$, respectively. The numerical simulations show that the parameter regime for simultaneous destabilization of the fixed point and stabilization of the target orbit, e.g., $N_{1}$, yields a global attractor basin including the whole area inside the UPO, while the stabilization of the UPO alone, e.g., at the point $N_{2}$, guarantees only local stability around the UPO. Note that the points of operation $N_{1}$ and $\mathrm{N}_{2}$ are inside and outside the primary region for the unstable fixed point, which corresponds to weakly and strongly UPOs, respectively, while $N_{3}$ indicates the point, where the period of the UPO becomes infinite. We note that, with the parameter set corresponding to the point $N_{2}$, the rotations around the fixed point and the UPO are in opposite direction of each other, since the angular frequency $\Omega_{0}=\omega_{0}-\gamma \lambda<0$ for $\omega_{0}=1>0$. In any case, the control signal vanishes when the target orbit is stabilized, demonstrating the noninvasiveness of our method.

It is worth noting that, in spite of the common belief that strongly unstable orbits are notoriously difficult to tackle by the conventional DFC, our control scheme allows us to extend remarkably the range of stabilization up to the point $C$, i.e., $\lambda^{*}$ determined by Eqs. (32) and (36) for linear and nonlinear control schemes, respectively. In particular, we see in Fig. $4 c$ that even orbits with infinite period can be stabilized. area inside the UPO. $\mathbf{b}$ The strongly unstable orbit with parameter set $N_{2}$ has the basin limited to a local neighborhood of the UPO. c Stabilization of the UPO with infinite period corresponding to point $N_{3}$. For every case, the control perturbations (gray curves) vanish when the system dynamics denoted by blue (dark) curves approach the target UPOs, demonstrating the noninvasiveness of our control scheme. Other parameters as in Fig. 1

\section{Delayed-feedback control with distributed delays}

In this section we study the stabilization of the UPO with distributed delayed feedback. This is common in a variety of systems where the delay times are given by a continuous distribution [48,52-54]. The model can be written as follows:

$$
\begin{aligned}
\dot{z}(t)= & {\left[\lambda+i \omega_{0}+(1+i \gamma)|z(t)|^{2}\right] z(t) } \\
& +K e^{i \beta} \int_{0}^{\infty} g(\tau)\left[e^{i \Omega_{0} \tau} z(t-\tau)-z(t)\right] d \tau,
\end{aligned}
$$

where $g(\tau)$ denotes a density distribution of time delays and is taken to be positive-definite and normalized to unity:

$g(\tau) \geq 0, \quad \int_{0}^{\infty} g(\tau) d \tau=1$.

In polar coordinates $z=r e^{i \varphi}$, Eq. (51) reads

$$
\begin{aligned}
\dot{r} & =\left(\lambda+r^{2}\right) r+K\left[\int _ { 0 } ^ { \infty } g ( \tau ) r ( t - \tau ) \operatorname { c o s } \left(\beta+\Omega_{0} \tau\right.\right. \\
& +\varphi(t-\tau)-\varphi) d \tau-r \cos \beta] \\
\dot{\varphi} & =\omega_{0}+\gamma r^{2}+K\left[\frac { 1 } { r } \int _ { 0 } ^ { \infty } g ( \tau ) r ( t - \tau ) \operatorname { s i n } \left(\beta+\Omega_{0} \tau\right.\right. \\
& +\varphi(t-\tau)-\varphi) d \tau-\sin \beta] .
\end{aligned}
$$


Obviously, Eqs. (51) and (52a, 52b) yield noninvasive solutions $r(t)=r_{0}$ and $\varphi(t)=\Omega_{0} t$ for any distribution function $g(\tau)$.

\subsection{Floquet exponents}

Using the ansatz $r(t)=r_{0}(1+\delta r(t)), \varphi(t)=\Omega_{0} t+\delta \varphi(t)$ and expanding Eqs. $(52 \mathrm{a}, 52 \mathrm{~b})$ to linear order in the small deviations $\delta r$ and $\delta \varphi$, we obtain

$$
\begin{aligned}
& \left(\begin{array}{l}
\dot{\delta r} \\
\dot{\delta} \varphi
\end{array}\right)=\left(\begin{array}{ll}
-2 \lambda & 0 \\
-2 \gamma \lambda & 0
\end{array}\right)\left(\begin{array}{l}
\delta r \\
\delta \varphi
\end{array}\right) \\
& \quad+K\left(\begin{array}{ll}
\cos \beta & -\sin \beta \\
\sin \beta & \cos \beta
\end{array}\right)\left(\begin{array}{l}
\int g(\tau) \delta r(t-\tau) d \tau-\delta r \\
\int g(\tau) \delta \varphi(t-\tau) d \tau-\delta \varphi
\end{array}\right),
\end{aligned}
$$

Using the exponential ansatz $(\delta r(t), \delta \varphi(t)) \propto \exp (\Lambda t)$ gives an equation for the Floquet exponents $\Lambda$ as follows:

$\operatorname{det}\left[\begin{array}{ll}-2 \lambda+K(\mathcal{G}(\Lambda)-1) \cos \beta-\Lambda & -K(\mathcal{G}(\Lambda)-1) \sin \beta \\ -2 \gamma \lambda+K(\mathcal{G}(\Lambda)-1) \sin \beta & K(\mathcal{G}(\Lambda)-1) \cos \beta-\Lambda\end{array}\right]=0$,

that is,

$$
\begin{aligned}
0= & \Lambda^{2}+2[\lambda-K(\mathcal{G}(\Lambda)-1) \cos \beta] \Lambda+K^{2}(\mathcal{G}(\Lambda)-1)^{2} \\
& -2 \lambda K(\mathcal{G}(\Lambda)-1)(\cos \beta+\gamma \sin \beta),
\end{aligned}
$$

where $\mathcal{G}(\Lambda)$ is the Laplace transform of the distribution function of delays, $g(\tau)$ :

$\mathcal{G}(\Lambda)=\int_{0}^{\infty} g(\tau) e^{-\Lambda \tau} d \tau$

\subsection{Enlargement of the stability region}

The dependence of the stability range on the distribution of delays is determined by Eq. (53), which is reduced to Eq. (27) with constant time delay for the case $g(u)=\delta(u-\tau)$.

Note that, if $g(\tau)$ has a sufficiently large variance, then $|\mathcal{G}(\Lambda)|$ is also sufficiently small, since the Laplace transform

$\mathcal{G}\left(\Lambda_{R}+i \Lambda_{I}\right)=\int_{0}^{\infty} g(\tau) e^{-\tau \Lambda_{R}}\left[\cos \left(\tau \Lambda_{I}\right)-i \sin \left(\tau \Lambda_{I}\right)\right] d \tau$

can be interpreted as a weighted average of the oscillatory integrand about zero over an interval determined by $g$. For the zero value of $\mathcal{G}(\Lambda)$ as an extreme case, Eq. (53) reduces to the algebraic equation $0=\Lambda^{2}+2(\lambda+K \cos \beta) \Lambda+$ $K^{2}+2 \lambda K(\cos \beta+\gamma \sin \beta)$, which yields the stabilization condition in a semi-open form as follows:

$K>-\lambda / \cos \beta$,
Thus, it is plausible that if the variance of the distribution is larger, the region of stabilization of the target orbit becomes larger.

To make further analytical progress, it is instructive to specify a particular choice of the delay distribution function. As the first example, we consider a uniform distribution

For uniform distribution with mean time delay $\langle\tau\rangle=\bar{\tau}$ and variance $\sigma^{2}=\rho^{2} / 3$, the delay kernel and the Laplace transform, respectively, are given by

$g(\tau)=\left\{\begin{array}{ll}\frac{1}{2 \rho} & \text { for } \bar{\tau}-\rho \leq \tau \leq \bar{\tau}+\rho, \\ 0 & \text { elsewhere. }\end{array}\right.$,

$\mathcal{G}(\Lambda ; \rho, \bar{\tau})=\frac{\sinh (\rho \Lambda)}{\rho \Lambda} e^{-\Lambda \bar{\tau}}$

As $\rho \rightarrow 0$, the relation $\mathcal{G}(\Lambda ; \rho, \bar{\tau}) \rightarrow e^{-\Lambda \bar{\tau}}$ holds, which recovers the discrete delay case. At a critical situation where the Hopf bifurcation of the UPO occurs, $\Lambda=i \Omega_{H}$, the Laplace transform is reduced to

$\mathcal{G}=\frac{\sin \left(\rho \Omega_{H}\right)}{\rho \Omega_{H}} e^{-i \Omega_{H} \bar{\tau}}$,

which becomes smaller as $\rho$ increases.

Figure 5 shows $\operatorname{Re}(\Lambda)$ in dependence on the coupling strength $K$ for uniform distribution of delays around $\bar{\tau}=$ $0.7 \pi$ and different values of $\rho$. We see that the curves for the transcritical mode (with zero imaginary part) show little difference between various values of $\rho$. The largest $\operatorname{Re} \Lambda$ for the Hopf mode (with non-zero imaginary part) shows that the

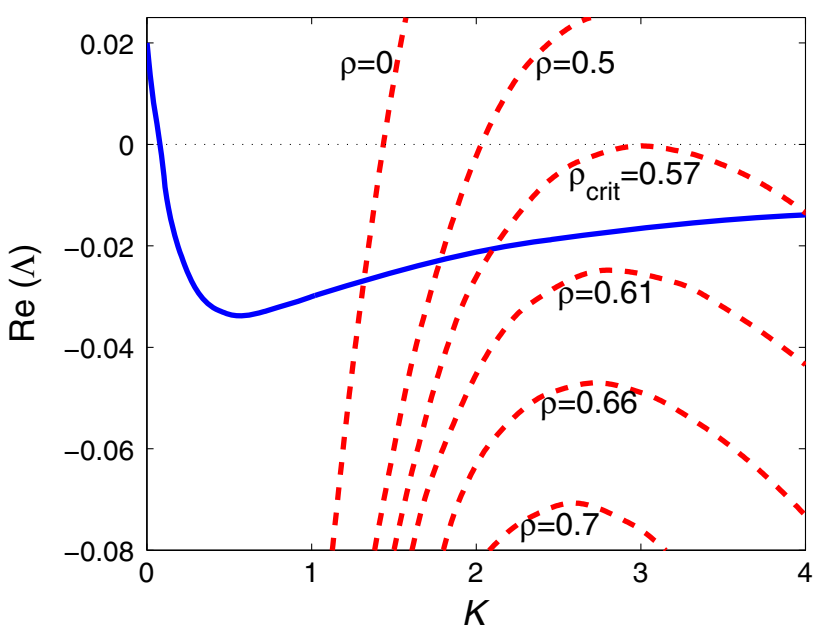

Fig. 5 (Color online) Real part of the Floquet exponents $\Lambda$ according to Eq. (53) with uniform distribution of delays versus the coupling strength $K$ for different values of half-width $\rho$. Parameters: $\lambda=-0.01, \bar{\tau}=$ $0.7 \pi$. The solid (blue) line denotes $\operatorname{Re} \Lambda$ for the transcritical mode, which shows little variation for different $\rho$. The dashed (red) lines indicate the largest $\operatorname{Re} \Lambda$ for the Hopf mode, which reveals an increase of the range of stability with increasing $\rho$. After a critical value, $\rho_{\text {crit }}=$ 0.57 , the stability region is determined only by the transcritical mode. Other parameters as in Fig. 1 
(a)

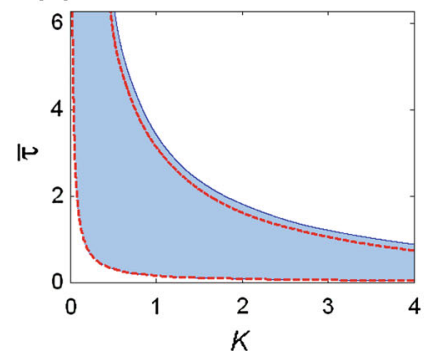

(b)

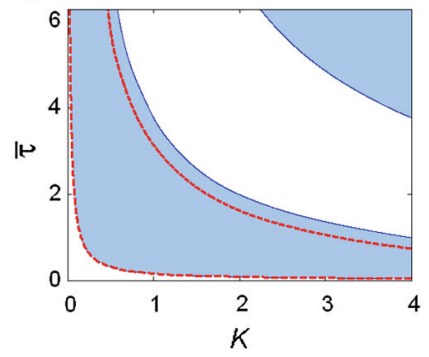

Fig. 6 (Color online) The stability region in the $(K, \bar{\tau})$ parameter plane for uniformly distributed delays: $\mathbf{a} \rho=0.33$, b $\rho=0.44, \mathbf{c} \rho=0.55$, and $\mathbf{d} \rho=0.66$. Other parameters as in Fig. 5. The area enclosed with (c)

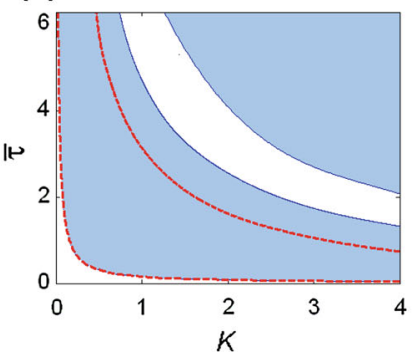

(d)

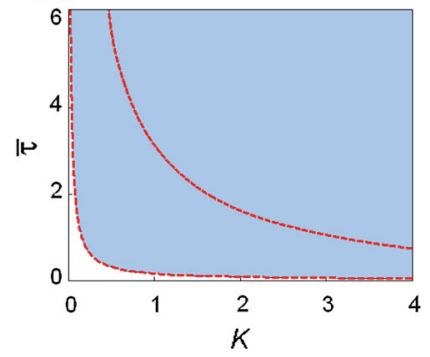

dotted (red) lines indicates the stable region for constant (discrete) delay. The stability region is greatly enlarged with increasing $\rho$ and, after a critical value, becomes bounded only by the transcritical curve

dynamics is described by

region of stabilization of the target orbit is greatly increase by the distributed delays. After a critical value, $\rho_{\text {crit }}=0.57$, the stability region is determined by the transcritical mode only.

The stability region in the $(K, \bar{\tau})$ parameter plane for uniformly distributed delays is shown in Fig. 6, where (a), (b), (c) and (d) corresponds to $\rho=0.33, \rho=0.44, \rho=0.55$, and $\rho=0.66$, respectively. Other parameters are the same as in Fig. 5. The area enclosed with dotted (red) lines indicates the stable region with constant (discrete) delay. Obviously, the stability region is greatly enlarged with increasing $\rho$ and becomes bounded only by the transcritical curve after a critical value.

For a Gamma distribution with mean time delay $\langle\tau\rangle=\frac{p}{\alpha}$ and variance $\sigma^{2}=\frac{p}{\alpha^{2}}$, the Laplace transform for $\alpha>0$ and the integer $p>0$ is given as

$g(\tau)=\frac{\alpha^{p} \tau^{p-1} e^{-\alpha \tau}}{(p-1) !}, \quad \mathcal{G}(\Lambda ; \alpha, p)=\frac{\alpha^{p}}{(\Lambda+\alpha)^{p}}$.

Note that for the Gamma distribution Eq. (53) reduces to a polynomial equation of $2 p+2$ degrees, rather than a transcendental one.

\section{Noninvasive control of synchrony in networks coupled with heterogeneous delays}

Our suggested control scheme with arbitrary delay-time lends itself for controlling the dynamics of a network coupled with heterogeneous delays. Also, it is possible to describe the supercritical as well as the subcritical case in a unified form.

\subsection{Model}

Consider delay-coupled networks of $N$ normal forms of a supercritical (-) or subcritical (+) Hopf bifurcation, whose

$$
\begin{aligned}
\dot{z}_{j}(t)= & {\left[\lambda+i \omega_{0} \mp(1+i \gamma)\left|z_{j}(t)\right|^{2}\right] z_{j}(t) } \\
& +K e^{i \beta} \sum_{n=1}^{N} a_{j n}\left[e^{i \psi_{j n}} z_{n}\left(t-\tau_{j n}\right)-z_{j}(t)\right],
\end{aligned}
$$

where $a_{j n}$ is the coupling matrix, and $j=1,2, \ldots, N$. We note that, unlike in our previous studies [20,44,45], the rotation angles $\psi_{j n}$ play the role of the control parameter, rather than the common coupling phase $\beta$. The parameters $\psi_{j n}$ essentially extend Sakaguchi's phase shift parameter $\alpha$ of two coupled phase oscillators [59]

$\dot{\theta_{1}}=\omega+K \sin \left(\theta_{2}-\theta_{1}-\alpha\right)$

$\dot{\theta}_{2}=\omega+K \sin \left(\theta_{1}-\theta_{2}-\alpha\right)$

to networks of Stuart-Landau oscillators. Note also that the interactions in Eq. (55) occur with heterogeneous delay times, $\tau_{j n}$.

Heterogeneous delays and the rotational coupling might give a natural way to model memory effects in interacting systems if we conceive the heterogeneous time delays and the rotation angle as proportional to the distance between nodes. If $a_{j j}=1$ for all $j$, this corresponds to a network with delayed self-feedback, while $a_{j j}=0$ characterizes a network without self-feedback.

Transforming Eq. (55) into amplitude and phase variables $r_{j}, \varphi_{j}$ using $z_{j}(t)=r_{j}(t) e^{i \varphi(t)}$, we obtain

$$
\begin{aligned}
\dot{r_{j}}= & \left(\lambda \mp r_{j}^{2}\right) r_{j}+K \sum_{n=1}^{N} a_{j n}\left[r _ { n } ( t - \tau _ { j n } ) \operatorname { c o s } \left(\beta+\psi_{j n}\right.\right. \\
& \left.\left.+\varphi_{n}\left(t-\tau_{j n}\right)-\varphi_{j}\right)-r_{j} \cos \beta\right] \\
\dot{\varphi_{j}}= & \omega_{0} \mp \gamma r_{j}^{2}+K \sum_{n=1}^{N} a_{j n}\left[\frac { r _ { n } ( t - \tau _ { j n } ) } { r _ { j } } \operatorname { s i n } \left(\beta+\psi_{j n}\right.\right. \\
& \left.\left.+\varphi_{n}\left(t-\tau_{j n}\right)-\varphi_{j}\right)-\sin \beta\right] .
\end{aligned}
$$


In the following, we focus on in-phase synchrony, splay states and clustering with a common amplitude $r_{j}=r_{0}$ and phases given by $\varphi_{j}(t)=\Omega t+j \Delta \phi_{m}$ with $\Delta \phi_{m}=$ $2 m \pi / N, m \in I$. The integer $m$ determines the specific state: in-phase oscillations correspond to $m=0$, while cluster and splay states correspond to $m=1, \ldots, N-1$. The cluster number $d_{c}$, which determines how many clusters of oscillators exist, is given by the least common multiple of $m$ and $N$ divided by $m$. Using abbreviation $\Phi_{n}^{j}=$ $\beta+\psi_{j n}-\Omega \tau_{j n}+(n-j) \Delta \phi_{m}$, we obtain the transcendental equation

$$
\begin{aligned}
& r_{0}^{2}= \pm\left\{\lambda-\mu K \cos \beta+K \sum_{n=1}^{N} a_{j n} \cos \Phi_{n}^{j}\right\}, \\
& \Omega=\omega_{0} \mp \gamma r_{0}^{2}-\mu K \sin \beta+K \sum_{n=1}^{N} a_{j n} \sin \Phi_{n}^{j},
\end{aligned}
$$

where a constant row sum of the coupling matrix $A$, i.e., $\sum_{n} a_{j n}=\mu$ is assumed, such that synchronized solutions exist.

Note that if $\Phi_{n}^{j}=\beta$, i.e., $\psi_{j n}=\Omega \tau_{j n}+(j-n) \Delta \phi_{m}$, Eqs. (58a, 58b) yields a unique solution

$r_{0}^{2}= \pm \lambda, \quad \Omega=\Omega_{0} \equiv \omega_{0}-\gamma \lambda$.

Thus, the multiple synchronous solutions resulting from the transcendental equations (58a, 58b) are eliminated by tuning

$\psi_{j n}=\Omega_{0} \tau_{j n}+(j-n) \Delta \phi_{m}$,

and the collective amplitude $r_{0}$ and frequency $\Omega$ of the synchronous states are given in a unique and noninvasive form, regardless of the network topology and the synchronization patterns.

\subsection{Calculation of Floquet exponents}

\subsubsection{Variational equations}

Using the ansatz $r_{j}(t)=r_{0}\left(1+\delta r_{j}(t)\right), \varphi_{j}(t)=\Omega t+$ $j \Delta \phi_{m}+\delta \varphi_{j}(t)$ and expanding Eqs. $(57 \mathrm{a}, 57 \mathrm{~b})$ to linear order in the small deviations $\delta r_{j}, \delta \varphi_{j}$ around the synchronous states we obtain

$$
\begin{aligned}
& \left(\begin{array}{l}
\delta \dot{r}_{j} \\
\delta \dot{\varphi}_{j}
\end{array}\right)=\left(\begin{array}{ll}
\mp 2 r_{0}^{2} & 0 \\
\mp 2 \gamma r_{0}^{2} & 0
\end{array}\right)\left(\begin{array}{l}
\delta r_{j} \\
\delta \varphi_{j}
\end{array}\right) \\
& +K \sum_{n} a_{j n}\left(\begin{array}{ll}
\cos \Phi_{n}^{j} & -\sin \Phi_{n}^{j} \\
\sin \Phi_{n}^{j} & \cos \Phi_{n}^{j}
\end{array}\right)\left(\begin{array}{l}
\delta r_{n}\left(t-\tau_{j n}\right)-\delta r_{j} \\
\delta \varphi_{n}\left(t-\tau_{j n}\right)-\delta \varphi_{j}
\end{array}\right)
\end{aligned}
$$

This is equivalent to

$\dot{\xi}_{j}=\mathbf{J}_{0}^{\mp} \xi_{j}+K \sum_{n} a_{j n} \mathbf{R}_{n}^{j}\left(\xi_{n}\left(t-\tau_{j n}\right)-\xi_{j}\right)$,

with $\xi_{j}=\left(\begin{array}{l}\delta r_{j} \\ \delta \varphi_{j}\end{array}\right), \quad \mathbf{J}_{0}^{\mp}=\left(\begin{array}{ll}\mp 2 r_{0}^{2} & 0 \\ \mp 2 \gamma r_{0}^{2} & 0\end{array}\right)$ and

$$
\mathbf{R}_{n}^{j}=\left(\begin{array}{l}
\cos \Phi_{n}^{j}-\sin \Phi_{n}^{j} \\
\sin \Phi_{n}^{j} \cos \Phi_{n}^{j}
\end{array}\right) \text {. }
$$

\subsubsection{Control by tuning the parameter $\psi_{j n}$}

When tuning by control equation (60) is applied, Eq. (61) is reduced to

$$
\begin{aligned}
\dot{\xi}_{j} & =\left(\mathbf{J}_{0}-K \mu \mathbf{R}\right) \xi_{j}+K \mathbf{R} \sum_{n} a_{j n} \xi_{n}\left(t-\tau_{j n}\right), \\
& =\left(\mathbf{J}_{0}-K \mu \mathbf{R}\right) \xi_{j}+K \mathbf{R} \sum_{n} a_{j n} e^{-\Lambda \tau_{j n}} \xi_{n},
\end{aligned}
$$

where $\mathbf{J}_{0}=\left(\begin{array}{ll}-2 \lambda & 0 \\ -2 \gamma \lambda & 0\end{array}\right)$ and $\mathbf{R}=\left(\begin{array}{ll}\cos \beta & -\sin \beta \\ \sin \beta & \cos \beta\end{array}\right)$. Here the relationship $\xi_{n}\left(t-\tau_{j n}\right)=e^{-\Lambda \tau_{j n}} \xi_{n}$ with Floquet exponents $\Lambda$ was used.

Note that Eq. (62) makes no distinction between super-and subcritical Hopf bifurcation and between the cluster numbers.

Using the notation $\mathbf{y}=\left(\xi_{1}, \xi_{2}, \ldots, \xi_{N},\right)^{T}$, Eq. (62) can be rewritten in matrix form as

$\dot{\mathbf{y}}=\left[\mathbf{I}_{\mathbf{N}} \otimes\left(\mathbf{J}_{\mathbf{0}}-K \mu \mathbf{R}\right)\right] \mathbf{y}+K(T \otimes \mathbf{R}) \mathbf{y}$,

where $I_{N}$ denotes the $N \times N$ identity matrix, and $T=$ $\left\{a_{j n} e^{-\Lambda \tau_{j n}}\right\}$.

If we assume that the matrix $T$ is diagonalized, the corresponding block-diagonalized equation is obtained in the decoupled form as follows:

$\dot{\zeta}_{k}(t)=\left[\mathbf{J}_{0}+K\left(\sigma_{k}-\mu\right) \mathbf{R}\right] \zeta_{k}(t)$,

where $\sigma_{k}$ denotes an eigenvalue of matrix $T$, and $k=$ $0,1, \ldots, N-1$.

The Floquet exponents of the synchronous periodic orbits are given by the eigenvalues $\Lambda$ of the characteristic equations as

$\operatorname{det}\left[\mathbf{J}_{\mathbf{0}}-\Lambda \mathbf{I}_{\mathbf{2}}+K\left(\sigma_{k}-\mu\right) \mathbf{R}\right]=0$.

Note that Eq. (64) defining the Floquet exponent $\Lambda$ depends on $\Lambda$ itself via $\sigma_{k}$. Nevertheless, the Floquet exponent $\Lambda$ can be formally expressed by the roots of the 2 nd degree polynomial

$\left|\begin{array}{cc}-2 \lambda+K\left(\sigma_{k}-\mu\right) \cos \beta-\Lambda & -K\left(\sigma_{k}-\mu\right) \sin \beta \\ -2 \gamma \lambda+K\left(\sigma_{k}-\mu\right) \sin \beta & K\left(\sigma_{k}-\mu\right) \cos \beta-\Lambda\end{array}\right|=0$, 
i.e.,

$$
\begin{aligned}
0= & \Lambda^{2}+2\left(\lambda-K \bar{Q}_{k} \cos \beta\right) \Lambda+K^{2} \bar{Q}_{k}^{2} \\
& -2 \lambda K \bar{Q}_{k}(\cos \beta+\gamma \sin \beta),
\end{aligned}
$$

with $\bar{Q}_{k}=\sigma_{k}-\mu$, which yields $\sigma_{k}$ as a function of Floquet exponents $\Lambda$ as follows:

$\sigma_{k}=s(\Lambda) \equiv \mu+\frac{1}{K}[\lambda(\cos \beta+\gamma \sin \beta)+\Lambda \cos \beta \pm \sqrt{D}]$.

where $D=\lambda^{2}\left(1+\gamma^{2}\right)-[\Lambda \sin \beta-\lambda(\gamma \cos \beta-\sin \beta)]^{2}$.

Finally, using Eq. (66), Floquet exponents $\Lambda$ for the Hopf normal form network coupled with heterogeneous delays can be determined in the self-consistent form by the transcendental equation

$\operatorname{det}\left[\begin{array}{c}a_{11} e^{-\Lambda \tau_{11}}-s(\Lambda) \\ a_{21} e^{-\Lambda \tau_{21}} \\ \vdots \\ a_{N 1} e^{-\Lambda \tau_{N 1}}\end{array}\right.$

$$
\begin{array}{cc}
a_{12} e^{-\Lambda \tau_{12}} & \cdots \\
a_{22} e^{-\Lambda \tau_{22}}-s(\Lambda) & \cdots \\
\vdots & \vdots \\
a_{N 2} e^{-\Lambda \tau_{N 2}} & \cdots
\end{array}
$$

If all Floquet exponents $\Lambda$ have negative real part for all $k$, the periodic orbits in the network are stabilized in a $m$ clustered state, i.e., a state of in-phase, splay, and cluster synchronization according to the choice of $m$ for the tuning parameter $\psi_{j n}$ given by Eq. (60).

We note that the characteristic equation for the Floquet exponents, Eq. (67), does not distinguish the bifurcation forms, i.e., the supercritical and subcritical case. Also, note that Eq. (67) determining the criteria for controlling the synchrony, does not include any parameters defining the synchronization patterns, e.g., the cluster number $m$. Therefore, the stability of the synchronous periodic orbits in the state of zero-lag, splay, and cluster is determined by the same equation, revealing the same parameter region.

\subsection{Special cases and numerical simulations}

\subsubsection{The case $\tau_{j n}=$ const.}

Due to the relation $T=e^{-\Lambda \tau} A$, the eigenvalue of matrix $T$ is given by $\sigma_{k}=v_{k} e^{-\Lambda \tau}$ with the eigenvalue $v_{k}$ of matrix $A=\left\{a_{j n}\right\}$. Then the master equation for the stability criteria for controlling the various synchrony patterns in both suband supercritical Hopf normal form networks is reduced to

$$
\begin{gathered}
\Lambda^{2}+2\left[\lambda-K Q_{k}(\Lambda) \cos \beta\right] \Lambda+K^{2} Q_{k}^{2}(\Lambda) \\
\quad-2 \lambda K Q_{k}(\Lambda)(\cos \beta+\gamma \sin \beta)=0,
\end{gathered}
$$

where $Q_{k}(\Lambda)=v_{k} e^{-\Lambda \tau}-\mu$. In the case that the delay time is adjusted to the UPO, Eq. (68) has been studied in [20,44,45], where stabilization of the UPOs via only in-phase synchronization for the subcritical case and controlling synchrony in the non-unique and invasive form for the supercritical case were investigated.

For $k=0$, corresponding to the synchronization manifold, Eq. (68) is reduced to Eq. (27) with the rescaled coupling strength $\bar{K}=\mu K$, since the relation $v_{0}=\mu$ holds for any network structure with a constant row sum $\mu$. The eigenvalues of coupling matrices $A$ without self-feedback and for $k \neq 0$ can be briefly summarized as follows: for all-to-all coupling, i.e., $a_{i j}=1$ for $i \neq j$ and zero otherwise, the eigenvalues are given by $v_{k}=-1$. Uni- and bidirectionally coupled rings yield $\nu_{k}=e^{2 i k \pi / N}$ and $\nu_{k}=2 \cos (2 \pi k / N)$,

$$
\left.\begin{array}{c}
a_{1 N} e^{-\Lambda \tau_{1 N}} \\
a_{2 N} e^{-\Lambda \tau_{2 N}} \\
\vdots \\
a_{N N} e^{-\Lambda \tau_{N N}}-s(\Lambda)
\end{array}\right]=0
$$

respectively. In the presence of self-feedback, a replacement $v_{k} \longmapsto v_{k}+1$ is needed.

For the supercritical case, we confine ourselves to $\beta=0$ and $\lambda>0$, then Eq. (68) is factorized:

$\left[\Lambda_{k}-K\left(v_{k} e^{-\Lambda \tau}-\mu\right)\right]\left[\Lambda_{k}-K\left(v_{k} e^{-\Lambda \tau}-\mu\right)+2 \lambda\right]=0$

which yields the dominant Floquet exponents as $\Lambda_{k}=$ $K\left(v_{k} e^{-\Lambda \tau}-\mu\right)$. It is obvious that these dominant Floquet exponents always have negative real part for any value of $K$ and $\tau$, for the various network structure (not only unidirectional ring but also bidirectional ring and all-to-all coupling). Thus, the $m$-clustering is stabilized in a unique and noninvasive form (59) for the general network topology by tuning the coupling rotation with Eq. (60), which is in a striking contrast to our previous studies $[44,45]$.

\subsubsection{Slightly nonidentical delay times}

When the time delays are distributed with small deviations around the average value, $\tau_{j n}=\bar{\tau}+\delta \tau_{j n}$, the matrix $T=$ $\left\{a_{j n} e^{-\Lambda \tau_{j n}}\right\}$ can be written as follows: 


$$
\begin{aligned}
& T=\left[\begin{array}{cccc}
a_{11} e^{-\Lambda\left(\bar{\tau}+\delta \tau_{11}\right)} & a_{12} e^{-\Lambda\left(\bar{\tau}+\delta \tau_{12}\right)} & \cdots & a_{1 N} e^{-\Lambda\left(\bar{\tau}+\delta \tau_{1 N}\right)} \\
a_{21} e^{-\Lambda\left(\bar{\tau}+\delta \tau_{21}\right)} & a_{22} e^{-\Lambda\left(\bar{\tau}+\delta \tau_{22}\right)} & \cdots & a_{2 N} e^{-\Lambda\left(\bar{\tau}+\delta \tau_{2 N}\right)} \\
\vdots & \vdots & \vdots & \vdots \\
a_{N 1} e^{-\Lambda\left(\bar{\tau}+\delta \tau_{N 1}\right)} & a_{N 2} e^{-\Lambda\left(\bar{\tau}+\delta \tau_{N 2}\right)} & \cdots & a_{N N} e^{-\Lambda\left(\bar{\tau}+\delta \tau_{N N}\right)}
\end{array}\right] \\
& \cong\left[\begin{array}{cccc}
a_{11} e^{-\Lambda \bar{\tau}}\left(1-\Lambda \delta \tau_{11}\right) & a_{12} e^{-\Lambda \bar{\tau}}\left(1-\Lambda \delta \tau_{12}\right) & \cdots & a_{1 N} e^{-\Lambda \bar{\tau}}\left(1-\Lambda \delta \tau_{1 N}\right) \\
a_{21} e^{-\Lambda \bar{\tau}}\left(1-\Lambda \delta \tau_{21}\right) & a_{22} e^{-\Lambda \bar{\tau}}\left(1-\Lambda \delta \tau_{22}\right) & \cdots & a_{2 N} e^{-\Lambda \bar{\tau}}\left(1-\Lambda \delta \tau_{2 N}\right) \\
\vdots & \vdots & \vdots & \vdots \\
a_{N 1} e^{-\Lambda \bar{\tau}}\left(1-\Lambda \delta \tau_{N 1}\right) & a_{N 2} e^{-\Lambda \bar{\tau}}\left(1-\Lambda \delta \tau_{N 2}\right) & \cdots & a_{N N} e^{-\Lambda \bar{\tau}}\left(1-\Lambda \delta \tau_{N N}\right)
\end{array}\right] \\
& =e^{-\Lambda \bar{\tau}}\left[\begin{array}{cccc}
a_{11} & a_{12} & \cdots & a_{1 N} \\
a_{21} & a_{22} & \cdots & a_{2 N} \\
\vdots & \vdots & \vdots & \vdots \\
a_{N 1} & a_{N 2} & \cdots & a_{N N}
\end{array}\right]-\Lambda e^{-\Lambda \bar{\tau}}\left[\begin{array}{cccc}
a_{11} \delta \tau_{11} & a_{12} \delta \tau_{12} & \cdots & a_{1 N} \delta \tau_{1 N} \\
a_{21} \delta \tau_{21} & a_{22} \delta \tau_{22} & \cdots & a_{2 N} \delta \tau_{2 N} \\
\vdots & \vdots & \vdots & \vdots \\
a_{N 1} \delta \tau_{N 1} & a_{N 2} \delta \tau_{N 2} & \cdots & a_{N N} \delta \tau_{N N}
\end{array}\right]=e^{-\Lambda \bar{\tau}}(A-\Lambda \delta T)
\end{aligned}
$$

where $\delta T=\left\{a_{j n} \delta \tau_{j n}\right\}$. That is, matrix $T$ is expressed by the sum of an average matrix and a perturbative matrix,

$T=T_{0}+\epsilon T_{1}$,

with $T_{0}=e^{-\Lambda \bar{\tau}} A$ and $\epsilon T_{1}=-\Lambda e^{-\Lambda \bar{\tau}} \delta T$. Since the eigenvalues of matrix $T_{0}$ are given by $\sigma_{k}=v_{k} e^{-\Lambda \bar{\tau}}$ with $v_{k}$, we expect that the eigenvalues of $T$ can be expressed by a sum of $v_{k}$ and the eigenvalues of $\epsilon T_{1}$, however, this point remains open.

\subsubsection{The case of $N=2$ with different delay times}

In the absence of self-feedback, Eq. (67) reads

$\operatorname{det}\left[\begin{array}{cc}-\sigma_{k} & e^{-\Lambda \tau_{12}} \\ e^{-\Lambda \tau_{21}} & -\sigma_{k}\end{array}\right]=0$,

which yields $\sigma_{k}= \pm e^{-\Lambda\left(\tau_{12}+\tau_{21}\right) / 2}$. Thus, the Floquet exponents are determined by

$$
\begin{aligned}
0= & \Lambda^{2}+2\left[\lambda-K Q_{ \pm}(\Lambda) \cos \beta\right] \Lambda+K^{2} Q_{ \pm}^{2}(\Lambda) \\
& -2 \lambda K Q_{ \pm}(\Lambda)(\cos \beta+\gamma \sin \beta),
\end{aligned}
$$

where $Q_{ \pm}(\Lambda)= \pm e^{-\Lambda \bar{\tau}}-1$ and $\bar{\tau}=\left(\tau_{12}+\tau_{21}\right) / 2$. Thus, the characteristic equation is dependent only on the mean value of the delay times, rather than $\tau_{12}$ and $\tau_{21}$. Equation (72) with sign ' + ' and ' - ' corresponds to the longitudinal and transversal modes, respectively. The former coincides with Eq. (27), and the latter was considered in our previous studies $([20,44,45])$. Note that Eq. (72) is independent of syn- chronization patterns, i.e., of both in-phase and anti-phase synchronization.

In the presence of self-feedback with $\tau_{11}=\tau_{22}=\tau_{0}$, Eq. (67), i.e.,

$\operatorname{det}\left[\begin{array}{cc}e^{-\Lambda \tau_{0}}-\sigma_{k} & e^{-\Lambda \tau_{12}} \\ e^{-\Lambda \tau_{21}} & e^{-\Lambda \tau_{0}}-\sigma_{k}\end{array}\right]=0$

yields $\sigma_{k}=e^{-\Lambda \tau_{0}} \pm e^{-\Lambda\left(\tau_{12}+\tau_{21}\right) / 2}$. If $\tau_{0}=\left(\tau_{12}+\tau_{21}\right) / 2$, then $\sigma_{0}=2 e^{-\Lambda \tau_{0}}$ and $\sigma_{1}=0$. Therefore, the longitudinal equation again coincides with Eq. (27) with the rescaled coupling strength, $\bar{K}=2 K$, while the transversal one is reduced to the following algebraic equation:

$$
\begin{gathered}
0=\Lambda^{2}+2(\lambda+K \mu \cos \beta) \Lambda+K^{2} \mu^{2} \\
+2 \lambda K \mu(\cos \beta+\gamma \sin \beta),
\end{gathered}
$$

which reveals readily the condition for synchronization of two oscillators as $K>-\lambda / \mu \cos \beta$.

\subsubsection{Numerical calculations}

Figure 7 shows time series from numerical simulations for four coupled subcritical Hopf normal forms in a bidirectional ring configuration with heterogeneous delays. The time series $\operatorname{Re}\left(z_{j}\right)$ in Fig. $7 \mathrm{a}-\mathrm{c}$ correspond to realization of in-phase synchronization with $\psi_{j n}=\Omega_{0} \tau_{j n}$, splay state with $\psi_{j n}=$ $\Omega_{0} \tau_{j n}+(j-n) \pi / 2$, and clustering with $\psi_{j n}=\Omega_{0} \tau_{j n}+$ $(j-n) \pi$, respectively, for $\lambda=-0.04$. In (d), it is shown that stabilization of four UPOs with infinite period for $\Omega_{0}=0$, 

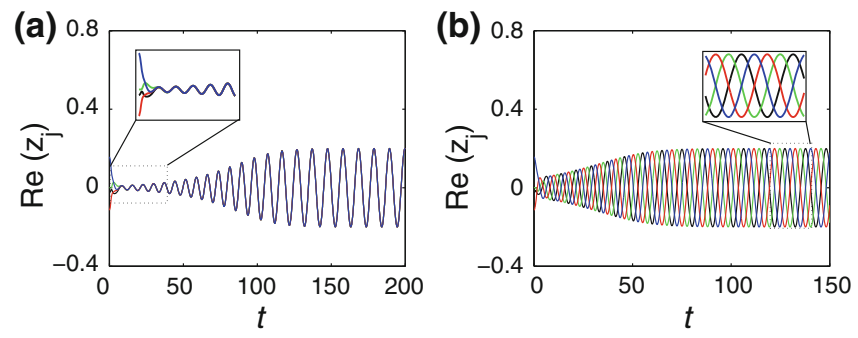

Fig. 7 (Color online) Controlling UPOs for a bidirectional ring coupled with heterogeneous delays for $N=4$. Heterogeneous delays were chosen as $\tau_{12}=0.78, \tau_{21}=0.66, \tau_{23}=0.94, \tau_{32}=0.6, \tau_{14}=$ $0.91, \tau_{41}=0.91, \tau_{34}=1.13, \tau_{43}=1.03$. Other parameters: $\omega_{0}=$ $1, \gamma=-10, \beta=\pi / 4$, and $K=0.3$. Time series $\operatorname{Re}\left(z_{j}\right)$ in a-c corre-

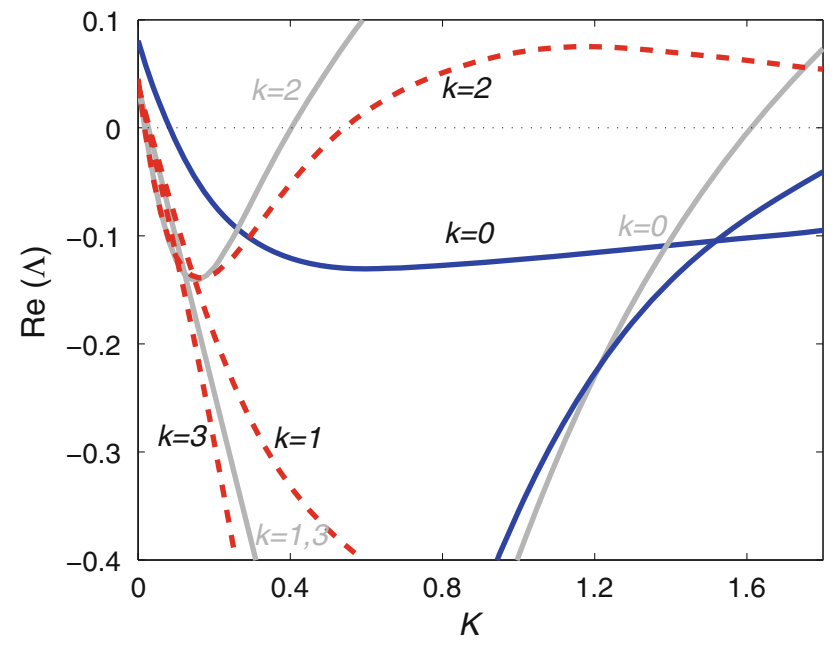

Fig. 8 (Color online) Real part of the leading Floquet exponents $\Lambda$ versus coupling strength $K$ in the bidirectionally coupled ring $(N=4)$ for the different mode numbers $k$. The solid(blue) line denotes $\operatorname{Re} \Lambda$ inside the synchronization manifold. The dashed (red) lines show the largest transversal $\operatorname{Re} \Lambda$ for different $k$. The solid gray line indicates the results for the discrete case, whose zero-crossing takes place ahead of the heterogeneous one. Parameters: $\lambda=-0.04, \tau_{12}=0.57, \tau_{21}=$ $1.45, \tau_{23}=1.19, \tau_{32}=0.66, \tau_{34}=0.69, \tau_{43}=0.38, \tau_{14}=$ $1.26, \tau_{41}=1.35$, and thus $\bar{\tau}=0.94$. Other parameters as Fig. 1

i.e., $\lambda=-0.1$ is also achieved in the splay state with $\psi_{j n}=$ $(j-n) \pi / 2$. Other parameters as in Fig. 1.

Figure 8 displays the real parts of the leading Floquet exponents $\Lambda$ as a function of $K$, determined by Eq. (67) for four subcritical Hopf normal forms in a bidirectional ring configuration with heterogeneous delays $\tau_{j n}$, as compared with the discrete delay fixed by the average delay $\bar{\tau}$ : $\tau_{12}=0.57, \tau_{21}=1.45, \tau_{23}=1.19, \tau_{32}=0.66, \tau_{34}=$ $0.69, \tau_{43}=0.38, \tau_{14}=1.26, \tau_{41}=1.35$, and thus $\bar{\tau}=$ 0.94. We took $\lambda=-0.04$ and others same as Fig. 1 .

Solid dark (blue) and dotted (red) curves correspond to $\operatorname{Re}(\Lambda)$ of the synchronization manifold and transversal modes for the heterogeneous delays, respectively. The gray curves denote the results for the discrete delays, determined
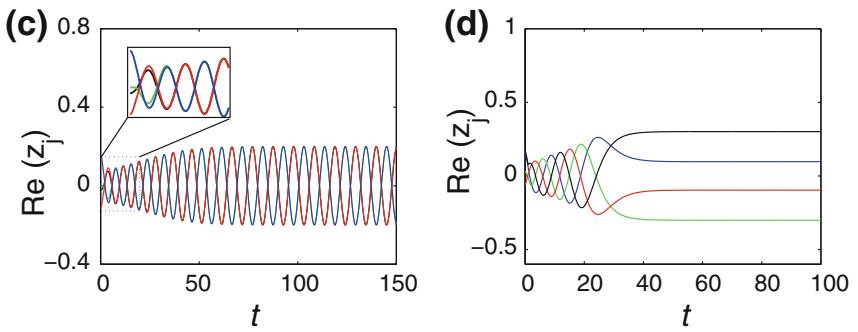

sponds to realization of in-phase synchronization with $\psi_{j n}=\Omega_{0} \tau_{j n}$, splay state with $\psi_{j n}=\Omega_{0} \tau_{j n}+(j-n) \pi / 2$, and clustering with $\psi_{j n}=\Omega_{0} \tau_{j n}+(j-n) \pi$, respectively, for $\lambda=-0.04$. d stabilization of four UPOs with infinite period $\Omega_{0}=0$, i.e., $\lambda=-0.1$ is achieved in splay state with $\psi_{j n}=(j-n) \pi / 2$

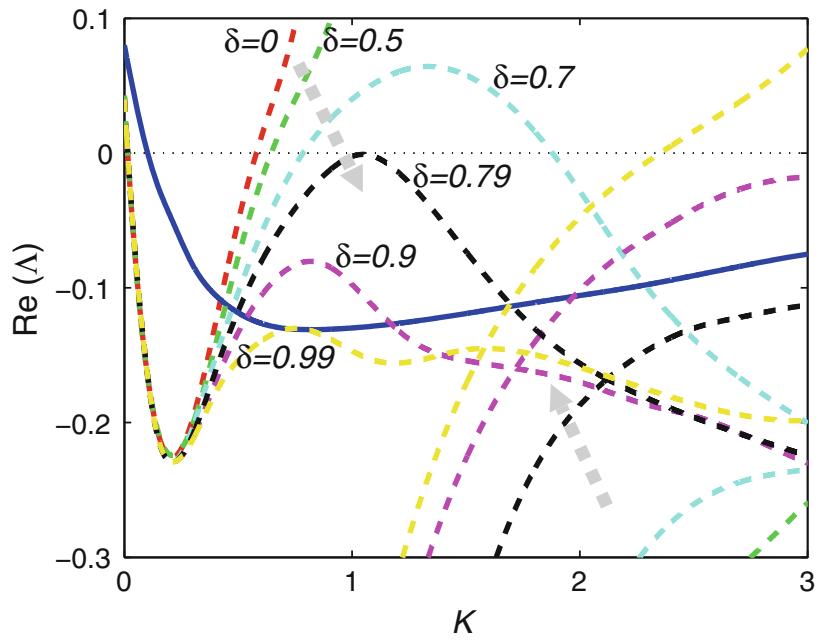

Fig. 9 (Color online) Real part of the leading Floquet exponents $\Lambda$ versus coupling strength $K$ in the bidirectionally coupled ring $(N=4)$ for the different $\delta$ that serves as a spread parameter in the heterogeneous delays $\tau_{j n}=\bar{\tau}\left(1+\delta \Xi_{j n}\right)$ with $\Xi_{j n}=$ $(0.64,0.32,-0.38,-0.42,-0.18,0.06,0.46,-0.5)$. Parameters: $\lambda=$ $-0.04, \bar{\tau}=0.94$, and others same as Fig. 1. The solid (blue) line denotes $\operatorname{Re} \Lambda$ inside the synchronization manifold. The dashed (red) lines show the largest transversal $\operatorname{Re} \Lambda$ for different $\delta$. The dashed arrows indicate the increasing direction of $\delta$

by Eq. (68). We see that there exists an interval of $K$ in which all of the real parts of Floquet exponents are negative. That is, the four UPOs are stabilized and the $m$-cluster is synchronized. Note that the interval for heterogeneous delay is larger than the discrete one. Furthermore, for $k=0$, the transcritical branch shows little difference for both the discrete and heterogeneous cases, while the Hopf branch for discrete delay becomes positive faster than the heterogeneous case. The transversal modes with $k=1$ and $k=3$ for the discrete delay configuration are overlapping since the bidirectional ring configuration yields the eigenvalues $v_{k}=2 \cos (2 \pi k / N)$ that are twice degenerate, while the heterogeneous delay configuration shows clearly separated lines for $k=1$ and $k=3$. 

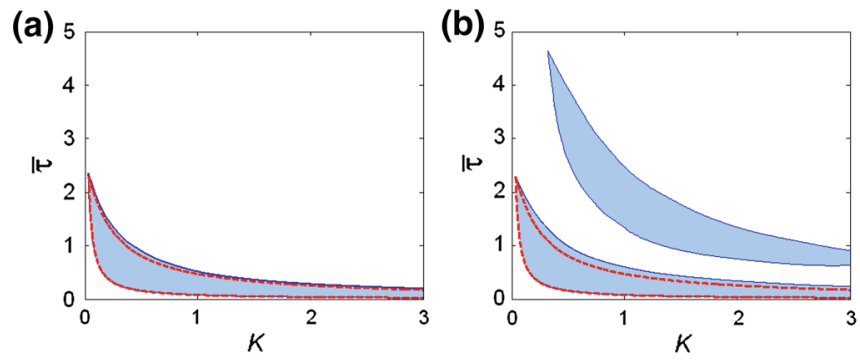

Fig. 10 (Color online) The stability region in the $(K, \bar{\tau})$ parameter plane for $N=4$ and heterogeneous delays: a $\delta=0.5, \mathbf{b} \delta=0.7$, c $\delta=0.9$, and $\mathbf{d} \delta=0.99$. Other parameters are the same as in Fig. 9 .

Figure 9 shows $\operatorname{Re}(\Lambda)$ in dependence on the coupling strength $K$ for different spread of heterogeneous delays around $\bar{\tau}=0.94$, determined by Eq. (67) for four subcritical Hopf normal forms in a bidirectional ring configuration. We assume that the delays are distributed according to $\tau_{j n}=\bar{\tau}\left(1+\delta \Xi_{j n}\right)$, where $\Xi_{j n} \in[-1,1]$ is random number with zero mean-value and $\delta$ serves as a parameter of spread of heterogeneous delays. For bidirectionally coupled ring of $N=4$, the eight random numbers were chosen as $\boldsymbol{\Xi}_{j n}=$ $(0.64,0.32,-0.38,-0.42,-0.18,0.06,0.46,-0.5)$ and $\delta$ is increased by $0,0.5,0.7,0.79,0.9,0.99$. Other parameters are the same as in Fig. 8. The solid dark (blue) curve corresponds to the modes longitudinal to the synchronization manifold with $k=0$, which exhibits little difference for different spread $\delta$. In contrast, the dotted curves corresponding to the transcritical branch for the transversal modes clearly show the larger descent with increasing $\delta$, which shows that even a small spread in the delay heterogeneity can greatly enlarge the set of parameters for which stabilization of the $m$-clustered UPOs can be achieved. We note that the Hopf branches for the transversal modes move in opposite direction with increasing $\rho$, as seen in the figure. Thus, the spread of heterogeneous delays can play a dual role in stabilizing periodic orbits: it can both facilitate the stabilization and lead to desychronization.

The stability region in the $(K, \bar{\tau})$ parameter plane in the bidirectional ring configuration for $N=4$ with heterogeneous delays for different values of $\delta$ is shown in Fig. 10, where (a), (b), (c), and (d) corresponds to $\delta=0.5, \delta=$ $0.7, \delta=0.9$, and $\delta=0.99$, respectively. The other parameters are the same as in Fig. 9. The area enclosed by dotted (red) lines indicates the stable region with discrete delay. Obviously, the stability region is enlarged with increasing $\delta$.

\subsection{Thermodynamic limit}

Now, we assume global coupling or all-to-all coupling, and let the number of elements in the ensemble tend to infinity. Moreover, time delays $\tau_{j n}$ are supposed to be distributed continuously with a density $g(\tau)$, independent of index $j$
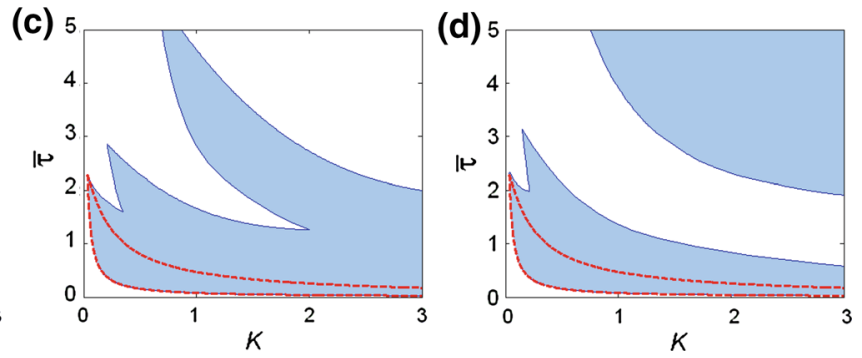

The area enclosed by dotted (red) lines indicates the stable region with discrete delay. The stability region is enlarged with increasing $\rho$

and $n$. This distribution function $g(\tau)$ is taken to be positive definite and normalized to unity:

$g(\tau) \geq 0, \quad \int_{0}^{\infty} g(\tau) d \tau=1$.

Then, one can apply the mean-field approximation to Eqs. (55) and (60) as follows:

$$
\begin{aligned}
\dot{z}_{j}(t)= & {\left[\lambda+i \omega_{0} \mp(1+i \gamma)\left|z_{j}(t)\right|^{2}\right] z_{j}(t) } \\
& +K e^{i \beta} \sum_{n=1}^{N} \bar{a}_{j n}\left[\int_{0}^{\infty} g(\tau) e^{i \psi_{j n}(\tau)} z_{n}(t-\tau) d \tau-z_{j}(t)\right],
\end{aligned}
$$

where $\psi_{j n}(\tau)=\Omega_{0} \tau+(j-n) \Delta \phi_{m}$, and $\bar{A}=\left\{\bar{a}_{j n}\right\}$ denotes the all-to-all coupling matrix.

The variational equations corresponding to Eq. (62) can be written as

$\dot{\xi}_{j}=\left(\mathbf{J}_{0}-K \mu \mathbf{R}\right) \xi_{j}+K \mathcal{G}(\Lambda) \mathbf{R} \sum_{n} \bar{a}_{j n} \xi_{n}$,

or in matrix form,

$\dot{\mathbf{y}}=\left[\mathrm{I}_{\mathbf{N}} \otimes\left(\mathbf{J}_{\mathbf{0}}-K \mu \mathbf{R}\right)\right] \mathbf{y}+K \mathcal{G}(\Lambda)(\bar{A} \otimes \mathbf{R}) \mathbf{y}$,

where

$\mathcal{G}(\Lambda)=\int_{0}^{\infty} g(\tau) e^{-\Lambda \tau} d \tau$

is the Laplace transform of the distribution function $g(\tau)$.

Eqs. (75) and (76) admit diagonalization, yielding the decoupled variational equations:

$\dot{\zeta}_{k}(t)=\left[\mathbf{J}_{0}+\mu K\left(\frac{\nu_{k}}{\mu} \mathcal{G}(\Lambda)-1\right) \mathbf{R}\right] \zeta_{k}(t)$,

where $k=0,1, \ldots, N-1$. The eigenvalue corresponding to the longitudinal mode is given by $\nu_{0}=\mu$. Note that, for $k \neq$ $0, v_{k}=0$ and $v_{k}=-1$ stands for all-to-all coupling with 
and without self-feedback, respectively. Thus, whether the self-feedback is included or not, has no influence on Eq. (78) for the transversal mode since $v_{k} / \mu \rightarrow 0$ as $\mu \rightarrow \infty$.

As a result, we obtain the characteristic equations for Floquet exponents for the longitudinal mode

$$
\begin{aligned}
0= & \Lambda^{2}+2[\lambda-\bar{K}(\mathcal{G}(\Lambda)-1) \cos \beta] \Lambda+\bar{K}^{2}(\mathcal{G}(\Lambda)-1)^{2} \\
& -2 \lambda \bar{K}(\mathcal{G}(\Lambda)-1)(\cos \beta+\gamma \sin \beta),
\end{aligned}
$$

and for the transversal mode

$0=\Lambda^{2}+2(\lambda+\bar{K} \cos \beta) \Lambda+\bar{K}^{2}+2 \lambda \bar{K}(\cos \beta+\gamma \sin \beta)$,

respectively, where $\bar{K}=\mu K$. The algebraic equation (80) readily reveals the condition for stable synchronization in the explicit form as follows

$\bar{K}>-\lambda / \cos \beta$,

which is independent of the feature of the delay distribution and has no upper boundary.

The dependence of the stability range on the distribution of heterogeneous delays is determined by Eq. (79). Note that Eq. (79) coincides with Eq. (53), the characteristic equation for the distributed-delay feedback control, except for the rescaled coupling strength $\bar{K}=\mu K$.

Note that, if $g(\tau)$ has a sufficiently large variance, then $|\mathcal{G}(\Lambda)|$ is also sufficiently small, since the Laplace transform

$\mathcal{G}\left(\Lambda_{R}+i \Lambda_{I}\right)=\int_{0}^{\infty} g(\tau) e^{-\tau \Lambda_{R}}\left[\cos \left(\tau \Lambda_{I}\right)-i \sin \left(\tau \Lambda_{I}\right)\right] d \tau$

can be interpreted as a weighted average of the oscillatory integrand about zero over an interval determined by $g$. For the zero value of $\mathcal{G}(\Lambda)$ as an extreme case, Eq. (79) reduces to the algebraic equation (80). Thus, it is plausible that if the variance of the distribution is larger, the region of stabilization of the target orbit becomes larger.

To make further analytical progress, it is instructive to specify a particular choice of the delay distribution function. As the first example, we consider a uniform distribution

$g(\tau)= \begin{cases}\frac{1}{2 \rho} & \text { for } \bar{\tau}-\rho \leq \tau \leq \bar{\tau}+\rho, \\ 0 & \text { elsewhere }\end{cases}$

This distribution has the mean time delay $\langle\tau\rangle=\bar{\tau}$, the variance $\sigma^{2}=\rho^{2} / 3$, and the Laplace transform

$\mathcal{G}(\Lambda ; \rho, \bar{\tau})=\frac{1}{2 \rho \Lambda} e^{-\Lambda \bar{\tau}}\left(e^{\rho \Lambda}-e^{-\rho \Lambda}\right)=\frac{\sinh (\rho \Lambda)}{\rho \Lambda} e^{-\Lambda \bar{\tau}}$.

As $\rho \rightarrow 0$, the relation $\mathcal{G}(\Lambda ; \rho, \bar{\tau})=e^{-\Lambda \bar{\tau}}$ holds, which corresponds to a discrete delay time. Figure 11 displays the real part of the leading Floquet exponents as a function of

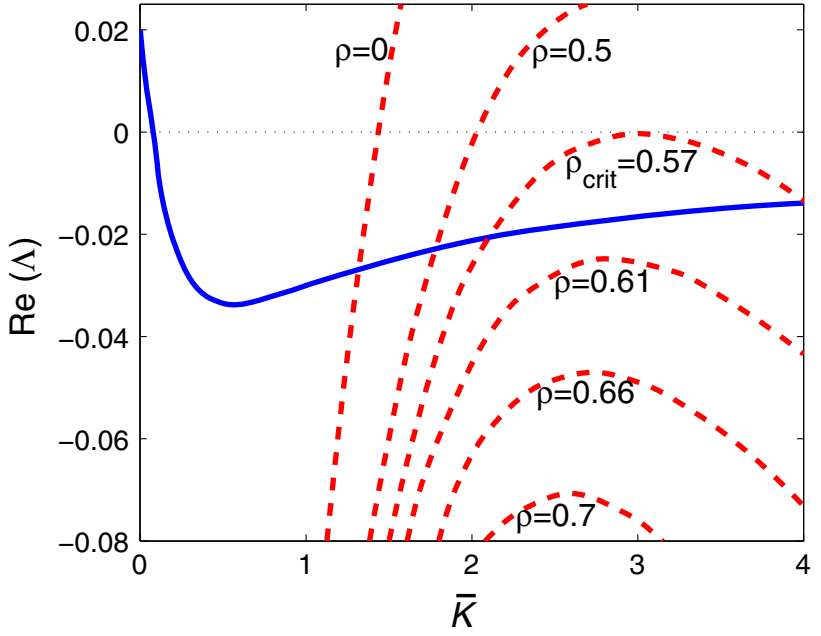

Fig. 11 (Color online) Real part of the Floquet exponents $\Lambda$ according to Eq. (79) with uniform distribution of delays versus the coupling strength $\bar{K}$ for different values of $\rho$. Parameters: $\lambda=-0.01, \bar{\tau}=$ $0.7 \pi$. The solid (blue) lines denotes $\operatorname{Re}(\Lambda)$ for the transcritical mode, which shows little difference for different values of $\rho$. The dashed (red) lines indicate the largest $\operatorname{Re}(\Lambda)$ for Hopf mode, which decreases with increasing $\rho$. After a critical value, $\rho_{\text {crit }}=0.57$, the stability region is determined only by the transcritical mode. Other parameters as in Fig. 1

$\bar{K}$ for different values of the variance $\rho$ of the heterogeneous delays around $\bar{\tau}=0.7 \pi$. We see that the transcritical mode that has only a real part, denoted with solid (blue) line, shows little difference between various values of $\rho$. On the other hand, the Hopf mode (red dashed) with non-zero imaginary part decreases with increasing $\rho$, which means that the region of stabilization is greatly enhanced by the heterogeneous delays. The stability area is increased with the variance of the uniform distribution, and at some critical value of the variance, $\rho_{\text {crit }}=0.57$, the real parts become all negative, and the stability region is determined by the transcritical mode only.

Figure 12 displays the stability region in the $(\bar{K}, \bar{\tau})$ parameter plane for different values of the variance. We observe clearly the enlarged parameter region for stabilization of the UPOs with increasing width of the heterogeneous delay distribution. Finally, a Gamma distribution of delays is considered as the second example:

$g(\tau)=\frac{\alpha^{p} \tau^{p-1} e^{-\alpha \tau}}{(p-1) !}$.

This distribution has the mean time delay $\langle\tau\rangle=\frac{p}{\alpha}$, the variance $\sigma^{2}=\frac{p}{\alpha^{2}}$, and the Laplace transform for $\alpha>0$ and the integer $p>0$

$\mathcal{G}(\Lambda ; \alpha, p)=\frac{\alpha^{p}}{(\Lambda+\alpha)^{p}}$.

Note that the Laplace transform of the Gamma distribution, Eq. (85), yields a polynomial characteristic equation of degree $2 p+2$, Eq. (79). 
(a)

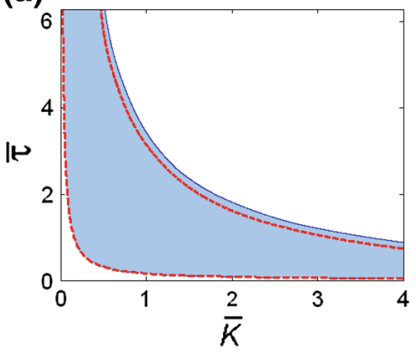

(b)

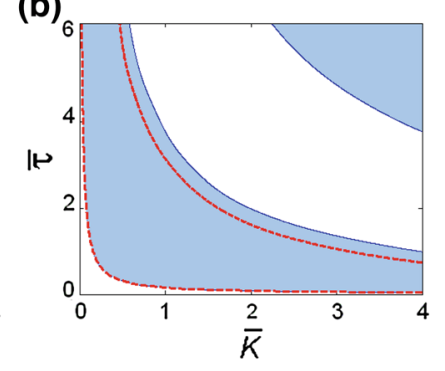

(c)

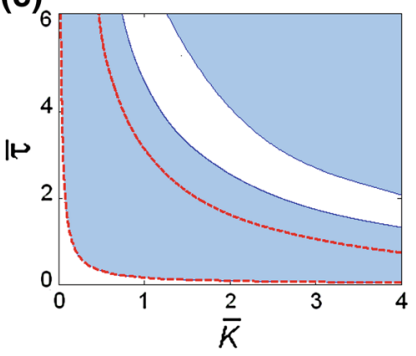

(d)

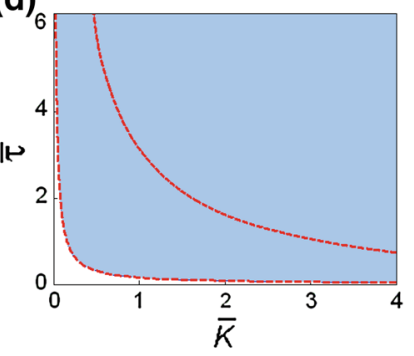

Fig. 12 (Color online) The stability region in the $(\bar{K}, \bar{\tau})$ parameter plane for uniformly distributed delays: a $\rho=0.33$, b $\rho=0.44$, c $\rho=0.55$, and $\mathbf{d} \rho=0.66$. Other parameters are the same as in Fig. 11 . The area enclosed by dotted (red) lines indicates the stable region with

\section{Conclusion}

We have proposed a delayed feedback control scheme with arbitrary delay for stabilizing the unstable periodic orbit in the normal form of a subcritical Hopf bifurcation.

In our control scheme, the constraint on the delay to be adjusted to the period of the target orbit is not imposed, while still retaining the condition of noninvasive control. Therefore, by including the delay as a variable bifurcation parameter, we have gained extensive insight into the bifurcation scenarios of the controlled dynamics: a complete bifurcation analysis for the fixed point as well as for the periodic orbit was presented, and the stability domains were identified. Not only weakly unstable periodic orbits but also strongly unstable orbits can be stabilized; the former have a global basin of attraction and the latter only a local basin. Even stabilization of the orbit with infinite period is achieved. A nonlinear control scheme has been developed and studied analytically, which has an advantage over the linear one, since the regime of stabilization of the target orbit is larger.

We have also studied the effects of distributed delayed feedback on the stabilization of the unstable periodic orbit, and we have shown that the regime of stabilization increases with increasing width of the distribution.

We have extended the control scheme to a network of Hopf normal forms coupled with heterogeneous delays. By tuning the parameters of coupling rotations, one of different synchronization patterns, i.e., in-phase, splay or clustering, can be chosen in a unique and noninvasive form, and their stabilizations can be efficiently achieved. The characteristic equation for the Floquet exponents of the heterogeneous delay network has been derived in an analytical form, which reveals the coupling parameters for successful stabilization. The equation takes a unified form regardless of the Hopf bifurcation forms including the subcritical and the supercritical case and regardless of the synchronization patterns. Analysis of Floquet exponents and direct numerical simulations have shown that the heterogeneity in delay makes the constant (discrete) delay. The stability region is greatly enlarged with increasing $\rho$ and, after a critical value, becomes bounded only by the transcritical curve

stabilization much easier, and provides an enlarged parameter region for successful control.

The thermodynamic limit of a network was considered in the framework of mean-field approximation, and the characteristic equations for the stability of UPOs was derived. Our results show that heterogeneous delays offer greatly enhanced performance for controlling periodic orbits.

From the point of view of applications, it looks very promising to apply our control scheme to semiconductor laser systems with arbitrary delay, i.e., control of UPOs and synchrony by tuning the feedback phase or coupling phase rather than the delay time.

Acknowledgments Chol-Ung Choe acknowledges support from Alexander von Humboldt Foundation. This work was also supported by DFG in the framework of Sfb 910. Philipp Hövel acknowledges support by BMBF (Grant No. 01Q1001B) in the framework of Bernstein Center for Computational Neuroscience Berlin.

\section{Appendix: Hopf normal-form reduction}

Here, we construct the normal form of Hopf bifurcation of the fixed point of Eq. (3) by using the method of multiple scales. We seek a solution of Eq. (3) in the form

$$
\begin{aligned}
z(t ; \epsilon)= & \epsilon z_{1}\left(T_{0}, T_{1}, T_{2}\right)+\epsilon^{2} z_{2}\left(T_{0}, T_{1}, T_{2}\right) \\
& +\epsilon^{3} z_{3}\left(T_{0}, T_{1}, T_{2}\right)+\cdots,
\end{aligned}
$$

where $T_{0}=t, T_{1}=\epsilon t, T_{2}=\epsilon^{2} t$, and $\epsilon$ is a bookkeeping parameter. The time derivative becomes

$\frac{d}{d t}=D_{0}+\epsilon D_{1}+\epsilon^{2} D_{2}+\cdots$

where $D_{i}=\partial / \partial T_{i}$. Thus,

$$
\begin{aligned}
\dot{z}= & \epsilon D_{0} z_{1}+\epsilon^{2}\left(D_{0} z_{2}+D_{1} z_{1}\right) \\
& +\epsilon^{3}\left(D_{0} z_{3}+D_{1} z_{2}+D_{2} z_{1}\right)+\cdots
\end{aligned}
$$


The delay term $z(t-\tau ; \epsilon)=\sum_{m=1}^{3} \epsilon^{m} z_{m}\left(T_{0}-\tau, T_{1}-\right.$ $\left.\epsilon \tau, T_{2}-\epsilon^{2} \tau\right)$. . becomes upon expansion for small $\epsilon$

$$
\begin{aligned}
z(t-\tau ; \epsilon)= & \epsilon z_{1 \tau}+\epsilon^{2}\left(z_{2 \tau}-\tau D_{1} z_{1 \tau}\right) \\
& +\epsilon^{3}\left(z_{3 \tau}-\tau D_{2} z_{1 \tau}-\tau D_{1} z_{2 \tau}\right),
\end{aligned}
$$

where $z_{i \tau}=z_{i}\left(T_{0}-\tau, T_{1}, T_{2}\right)$. Substituting Eqs. (86)-(89) into Eq. (3) and equating coefficients of equal powers of $\epsilon$, we obtain

$$
\begin{aligned}
O(1): D_{0} z_{1}= & \left(\lambda+i \omega_{0}\right) z_{1}+K e^{i \beta}\left(e^{i \Omega_{0} \tau} z_{1 \tau}-z_{1}\right), \\
O(2): D_{0} z_{2}= & \left(\lambda+i \omega_{0}\right) z_{2} \\
& +K e^{i \beta}\left[e^{i \Omega_{0} \tau}\left(z_{2 \tau}-\tau D_{1} z_{1 \tau}\right)-z_{2}\right]-D_{1} z_{1},
\end{aligned}
$$

$$
\begin{aligned}
O(3): D_{0} z_{3}= & \left(\lambda+i \omega_{0}\right) z_{3}+K e^{i \beta}\left[e ^ { i \Omega _ { 0 } \tau } \left(z_{3 \tau}\right.\right. \\
& \left.\left.-\tau D_{2} z_{1}\right)-z_{3}\right]-D_{2} z_{1}+(1+i \gamma)\left|z_{1}\right|^{2} z_{1}
\end{aligned}
$$

The general solution of Eq. (90) can be expressed as

$z_{1}=A\left(T_{1}, T_{2}\right) e^{i \omega_{H} T_{0}}+\sum_{m=1}^{\infty} A_{m}\left(T_{1}, T_{2}\right) e^{\left(\sigma_{m}+i \omega_{m}\right) T_{0}}$,

where $\omega_{H}$ is the critical frequency corresponding to a Hopf bifurcation of the fixed point and $\sigma_{m}+i \omega_{m}$ are the remaining roots of Eq. (7). Near the stability boundary, all of the eigenvalues have negative real parts except the eigenvalue corresponding to $\omega_{H}$. As time increases all terms in Eq. (93) decay with time, leaving only the first term:

$z_{1}=A\left(T_{1}, T_{2}\right) e^{i \omega_{H} T_{0}}$,

which gives $z_{1 \tau}=e^{i \omega_{H} \tau} z_{1}$. Substituting Eq. (94) into (91) and eliminating the source of secular terms, we have

$\left[1+K \tau e^{i\left(\beta+\Omega_{0} \tau-\omega_{H} \tau\right)}\right] D_{1} z_{1}=0$ or $A=A\left(T_{2}\right)$.

Since $z_{1}$ is independent of $T_{1}$, the higher-order terms in Eq. (86) are also independent of this time scale. Substituting Eqs. (95) into (92) and eliminating the terms that produce secular terms, we obtain the normal form of Hopf bifurcation as follows as

$A^{\prime}=\frac{1+i \gamma}{1+K \tau e^{i\left(\beta+\Omega_{0} \tau-\omega_{H} \tau\right)}}|A|^{2} A$.

The condition on the sign of the real part of the cubic coefficient, determining the type of Hopf bifurcation, results in Eq. (24).

\section{References}

1. Pyragas K (1992) Continuous control of chaos by self-controlling feedback. Phys Lett A 170:421-428
2. Pyragas K (2006) Delayed feedback control of chaos. Philos Trans R Soc A 364:2309-2334

3. Schöll E, Schuster HG (2008) Handbook of chaos control. Wiley$\mathrm{VCH}$, Weinheim (second completely revised and enlarged edition)

4. Just W, Pelster A, Schanz M, Schöll E (2010) Delayed complex systems. Philos Trans R Soc A 368:301-513

5. Flunkert V, Fischer I, Schöll E (2013) Dynamics, control and information in delay-coupled systems. Philos Trans R Soc A 371:20120465-1-20120465-4

6. Nakajima H (1997) On analytical properties of delayed feedback control of chaos. Phys Lett A 232:201-207

7. Schuster HG, Stemmler MB (1997) Control of chaos by oscillating feedback. Phys Rev E 56:6410-6417

8. Nakajima H, Ueda Y (1998) Limitation of generalized delayed feedback control. Phys D 111:143-150

9. Pyragas V, Pyragas K (2006) Delayed feedback control of the Lorenz system: an analytical treatment at a subcritical Hopf bifurcation. Phys Rev E 73:036215-1-036215-10

10. Pyragas K (2001) Control of chaos via an unstable delayed feedback controller. Phys Rev Lett 86:2265-2268

11. Choe CU, Flunkert V, Hövel P, Benner H, Schöll E (2007) Conversion of stability in systems close to a Hopf bifurcation by timedelayed coupling. Phys Rev E 75:046206-1-046206-7

12. Fiedler B, Flunkert V, Georgi M, Hövel P, Schöll E (2007) Refuting the odd number limitation of time-delayed feedback control. Phys Rev Lett 98:114101-1-114101-4

13. von Loewenich C, Benner H, Just W (2010) Experimental verification of Pyragas-Schöll-Fiedler control. Phys Rev E 82:036204$1-036204-6$

14. Schikora S, Wünsche HJ, Henneberger F (2011) Odd-number theorem: optical feedback control at a subcritical Hopf bifurcation in a semiconductor laser. Phys Rev E 83:026203-1-026203-7

15. Hooton EW, Amann A (2012) Analytical limitation for timedelayed feedback control in autonomous systems. Phys Rev Lett 109:154101-1-154101-5

16. Amann A, Hooton EW (2013) An odd-number limitation of extended time-delayed feedback control in autonomous systems. Philos Trans R Soc A 371:20120463-1-20120463-8

17. Just W, Fiedler B, Flunkert V, Georgi M, Hövel P, Schöll E (2007) Beyond odd number limitation: a bifurcation analysis of timedelayed feedback control. Phys Rev E 76:026210-1-026210-11

18. Postlethwaite CM, Silber M (2007) Stabilizing unstable periodic orbits in the Lorenz equations using time-delayed feedback control. Phys Rev E 76:056214-1-056214-10

19. Brown G, Postlethwaite CM, Silber M (2011) Time-delayed feedback control of unstable periodic orbits near a subcritical Hopf bifurcation. Phys D 240:859-871

20. Choe CU, Jang H, Flunkert V, Dahms T, Hövel P, Schöll E (2013) Stabilization of periodic orbits near a subcritical Hopf bifurcation in delay-coupled networks. Dyn Syst 28:15-33

21. Watts DJ, Strogatz SH (1998) Collective dynamics of 'small-world' networks. Nature 393:440-442

22. Albert R, Barabási A-L (2002) Statistical mechanics of complex networks. Rev Mod Phys 74:47-97

23. Newman MEJ (2003) The structure and function of complex networks. SIAM Rev 45:167-256

24. Barnat AV, Lu T-M (2003) Pulsed and pulsed bias sputtering: principles and applications. Kluwer Academic, Boston

25. Pikovsky AS, Rosenblum MG, Kurths J (2001) Synchronization: a universal concept in nonlinear sciences. Cambridge University Press, Cambridge

26. Boccaletti S, Bragard J (2006) Controlling spatio-temporal chaos in the scenario of the one-dimensional complex Ginzburg-Landau equation. Philos Trans R Soc (Lond) A 364:2383-2395

27. Yeung MKS, Strogatz SH (1999) Time delay in the Kuramoto model of coupled oscillators. Phys Rev Lett 82:648-651 
28. Earl MG, Strogatz SH (2003) Synchronization in oscillator networks with delayed coupling: a stability criterion. Phys Lett E 67:036204-1-036204-4

29. Dhamala M, Jirsa VK, Ding M (2004) Enhancement of neural synchrony by time delay. Phys Rev Lett 92:074104-1-074104-4

30. Yanchuk S, Wolfrum M, Hövel P, Schöll E (2006) Control of unstable steady states by long delay feedback. Phys Rev E 74:026201$1-026201-7$

31. Fischer I, Vicente R, Buldú JM, Peil M, Mirasso CR, Torrent MC, García-Ojalvo J (2006) Zero-lag long-range synchronization via dynamical relaying. Phys Rev Lett 97:123902-1-123902-4

32. Flunkert V, D'Huys O, Danckaert J, Fischer I, Schöll E (2009) Bubbling in delay-coupled lasers. Phys Rev E 79:065201-1-065201-4

33. Kinzel W, Englert A, Reents G, Zigzag M, Kanter I (2009) Synchronization of networks of chaotic units with time-delayed couplings. Phys Rev E 79:056207-1-056207-4

34. Fiedler B, Flunkert V, Hövel P, Schöll E (2010) Beyond the odd number limitation of time-delayed feedback control of periodic orbits. Eur Phys J Special Top 191:53-70

35. Schöll E (2010) Pattern formation and time-delayed feedback control at the nano-scale. In: Radons G, Rumpf B, Schuster HG (eds) Nonlinear dynamics of nanosystems. Wiley- $\mathrm{VCH}$, Weinheim, pp 325-367

36. Flunkert V, Yanchuk S, Dahms T, Schöll E (2010) Synchronizing distant nodes: a universal classification of networks. Phys Rev Lett 105:254101-1-254101-4

37. Heiligenthal S, Dahms T, Yanchuk S, Jüngling T, Flunkert V, Kanter I, Schöll E, Kinzel W (2011) Strong and weak chaos in nonlinear networks with time-delayed couplings. Phys Rev Lett 107:234102$1-234102-5$

38. Lehnert J, Dahms T, Hövel P, Schöll E (2011) Loss of synchronization in complex neural networks with delay. Eur Phys Lett 96:60013-p1-1-60013-p6

39. Keane A, Dahms T, Lehnert J, Suryanarayana SA, Hövel P, Schöll E (2012) Synchronisation in networks of delay-coupled type-I excitable systems. Eur Phys J B 85:407

40. Dahms T, Lehnert J, Schöll E (2012) Cluster and group synchronization in delay-coupled networks. Phys Rev E 86:016202-1016202-10

41. Selivanov AA, Lehnert J, Dahms T, Hövel P, Fradkov AL, Schöll E (2012) Adaptive synchronization in delay-coupled networks of Stuart-Landau oscillators. Phys Rev E 85:016201-1-016201-8

42. Schöll E, Selivanov AA, Lehnert J, Dahms T, Hövel P, Fradkov AL (2012) Control of synchronization in delay-coupled networks. Int J Mod Phys B 26:1246007-1-1246007-11

43. Schöll E (2013) Synchronization in delay-coupled complex networks. In: J-Q Sun, Q Ding (eds) Advances in analysis and control of time-delayed dynamical systems, chap 4. World Scientific, Singapore, pp 57-83
44. Choe CU, Dahms T, Hövel P, Schöll E (2010) Controlling synchrony by delay coupling in networks: from in-phase to splay and cluster states. Phys Rev E 81:025205-1-025205-4

45. Choe CU, Dahms T, Hövel P, Schöll E (2011) Control of synchrony by delay coupling in complex networks. In: Proceedings of the eighth AIMS international conference on dynamical systems, differential equations and applications. American Institute of Mathematical Sciences, Springfield, pp 292-301

46. Cushing JM (1977) Integrodifferential equations and delay models in population dynamics, vol 20. Springer, Heidelberg

47. Masoller C, Martí AC (2005) Random delays and the synchronization of chaotic maps. Phys Rev Lett 94:134102-1-134102-4

48. Vicente R, Gollo LL, Mirasso CR, Fischer I, Gordon P (2008) Dynamical relaying can yield zero time lag neuronal synchrony despite long conduction delays. Proc Natl Acad Sci USA 105:17157

49. Panchuk A, Rosin DP, Hövel P, Schöll E (2013) Synchronization of coupled neural oscillators with heterogeneous delays. Int J Bifurc Chaos 23:1330039-1-1330039-15

50. Cakan C, Lehnert J, Schöll E (2014) Heterogeneous delays in neural networks. Eur Phys J B, in print (available as arXiv:1311.1919v1)

51. Atay FM (2003) Distributed delays facilitate amplitude death of coupled oscillators. Phys Rev Lett 91:094101-1-094101-4

52. Kyrychko YN, Blyuss KB, Schöll E (2011) Amplitude death in systems of coupled oscillators with distributed-delay coupling. Eur Phys J B 84:307-315

53. Kyrychko YN, Blyuss KB, Schöll E (2013) Amplitude and phase dynamics in oscillators with distributed-delay coupling. Philos Trans R Soc A 371:20120466-1-20120466-22

54. Gjurchinovski A, Jüngling T, Urumov V, Schöll E (2013) Delayed feedback control of unstable steady states with high-frequency modulation of the delay. Phys Rev E 88:032912-1-032912-14

55. Schneider I (2013) Delayed feedback control of three diffusively coupled Stuart-Landau oscillators: a case study in equivariant Hopf bifurcation. Philos Trans R Soc A 371:20120472-1-20120472-10

56. Postlethwaite CM, Brown G, Silber M (2013) Feedback control of unstable periodic orbits in equivariant Hopf bifurcation problems. Philos Trans R Soc A 371:20120467-1-20120467-20

57. Fiedler B (1988) Global bifurcation of periodic solutions with symmetry. Springer, Heidelberg

58. Golubitsky M, Stewart I (2002) The symmetry perspective. Birkhäuser, Basel

59. Sakaguchi H (1988) Cooperative phenomena in coupled oscillator systems under external fields. Prog Theor Phys 79:39-46 